\title{
CONTRACTING ENDOMORPHISMS AND DUALIZING COMPLEXES
}

\author{
SAEED NASSEH AND SEAN SATHER-WAGSTAFF
}

\begin{abstract}
We investigate how one can detect the dualizing property for a chain complex over a commutative local noetherian ring $R$. Our focus is on homological properties of contracting endomorphisms of $R$, e.g., the Frobenius endomorphism when $R$ contains a field of positive characteristic.
\end{abstract}

\section{INTRODUCTION}

Throughout this paper, the term "ring" means "commutative noetherian ring with identity", and "module" means "unital module". A ring is "complete" if it is complete (i.e., separated and complete) with respect to its Jacobson radical. Let $R$ be a ring. For this section, assume that $(R, \mathfrak{m}, k)$ is local.

An idea in commutative algebra that is now standard is the following: interesting properties of $R$ can be detected by homological conditions on $k$; when $R$ contains a field of positive characteristic, such properties of $R$ can be detected similarly by ${ }^{n} R$. Here ${ }^{n} R$ is the additive abelian group $R$ viewed as an $R$-module via restriction of scalars along the $n$th iterated Frobenius map $f_{R}^{n}: R \rightarrow R$ given by $r \mapsto r^{p^{n}}$.

The somewhat canonical example of this is Auslander, Buchsbaum, Kunz, Rodicio, and Serre's work 3, 24, 29, 32 characterizing regular rings in terms of finite projective dimension of $k$ and finite flat dimension of ${ }^{n} R$. Analogous characterizations of the Gorenstein property are built from Auslander and Bridger's G-dimension 2] (or using similar ideas) by Goto, Iyengar, Sather-Wagstaff, Takahashi, and Yoshino [19, 23, 33].

A comparable characterization of the dualizing property for $R$-complexes in terms of derived reflexive behavior of $k$ goes back to Hartshorne and Grothendieck [21. The point of this paper is to give similar characterizations of dualizing complexes with respect to ${ }^{n} R$. We frame the conversation in terms of Christensen's semidualizing complexes [11] (coming from Avramov and Foxby's relative dualizing complexes [6]), and following Avramov, Iyengar, and Miller [9] in terms of contracting endomorphisms. (See Section 1 for terminology and background results.) A special case of one of our main results is the following, which we prove in 3.4

Theorem A. Let $\varphi: R \rightarrow R$ be a module-finite contracting endomorphism, and let $C$ be a semidualizing $R$-complex. Let ${ }^{n} R$ be the additive abelian group $R$ viewed as an $R$-module via restriction of scalars along the $n$-fold composition $\varphi^{n}: R \rightarrow R$. Then the following conditions are equivalent:

2010 Mathematics Subject Classification. 13A35, 13D05, 13D09.

Key words and phrases. Bass classes, contracting endomorphisms, dualizing complexes, $\mathrm{G}_{C^{-}}$ dimensions, Frobenius endomorphisms, semidualizing complexes.

This material is based on work supported by North Dakota EPSCoR and NSF Grant EPS0814442. Sather-Wagstaff was supported in part by a grant from the NSA. 
(i) $C$ is a dualizing $R$-complex.

(ii) $C \sim \mathbf{R H o m}_{R}\left({ }^{n} R, C\right)$ for some $n>0$.

(iii) $\mathrm{G}_{C}$-dim ${ }^{n} R<\infty$ and $C$ is derived $\mathbf{R} \operatorname{Hom}_{R}\left({ }^{n} R, C\right)$-reflexive for some $n>0$.

(iv) $\mathrm{G}_{C}$-dim ${ }^{n} R<\infty$ for infinitely many $n>0$.

If $R$ has a dualizing complex $D$, then these conditions are equivalent to the following:

(v) $\mathrm{G}_{C}$ - $\operatorname{dim}{ }^{n} R<\infty$ and ${ }^{n} R \otimes_{R}^{\mathbf{L}} \mathbf{R} \operatorname{Hom}_{R}(C, D)$ is derived $\mathbf{R} \operatorname{Hom}_{R}(C, D)$-reflexive for some $n>0$.

A standard technique for working with the Frobenius involves reducing to the case where $R$ is $F$-finite. The next result shows how this works in our setting; it is contained in Theorem 4.2 .

Theorem B. Let $R$ be a local ring of prime characteristic $p>0$, and let $C$ be a semidualizing $R$-complex. Then the following conditions are equivalent:

(i) $C$ is a dualizing $R$-complex.

(ii) There is a complete weakly étale $F$-finite local $R$-algebra $S$ such that $S \otimes_{R}^{\mathbf{L}} C$ is dualizing for $S$.

(iii) There is a complete weakly étale $F$-finite local $R$-algebra $S$ such that for infinitely many $n>0$ one has $\mathrm{G}_{S \otimes_{R} \mathrm{~L} C}-\operatorname{dim} f_{S}^{n}<\infty$.

(iv) There is a complete weakly étale $F$-finite local $R$-algebra $S$ such that for some $n>0$ one has $\mathrm{G}_{S \otimes C_{R}}-\operatorname{dim} f_{S}^{n}<\infty$ and ${ }^{n} S \otimes_{S}^{L} \mathbf{R} \operatorname{Hom}_{S}\left(S \otimes{ }_{R}^{\mathbf{L}} C, D^{S}\right)$ is derived $\mathbf{R H o m}_{S}\left(S \otimes_{R}^{\mathbf{L}} C, D^{S}\right)$-reflexive, where $D^{S}$ is a dualizing $S$-complex.

It is worth noting that one of the focuses of this paper involves developing a similar method for reducing to the module-finite situation for other contracting endomorphisms.

We conclude this section by summarizing the contents of the paper. Section 1 contains terminology and background content. Section 2 consists of analyses of a construction like $\mathbf{R H o m}\left({ }^{n} R, C\right)$ that is better suited for endomorphisms that are not module-finite. In Section 3 we prove results including Theorem $\mathrm{A}$ above about general contracting endomorphisms, and in Section 4 we focus briefly on the Frobenius endomorphism. Finally, Appendix A contains a somewhat general construction of module-finite contracting endomorphisms.

\section{Semidualizing complexes and $\mathrm{G}_{C}$-Dimension}

In this section, we recall definitions and background material on semidualizing complexes and related notions. We begin by specifying our notation for complexes and derived categories. The reader may find [18, 21, 34, 35] to be useful for more background.

1.1. In this paper, $R$-complexes are indexed homologically

$$
M=\cdots \stackrel{\partial_{i+1}^{M}}{\longrightarrow} M_{i} \stackrel{\partial_{i}^{M}}{\longrightarrow} M_{i-1} \stackrel{\partial_{i-1}^{M}}{\longrightarrow} \cdots .
$$

For each integer $i$, the $i$ th suspension (or shift) of $M$, denoted $\Sigma^{i} M$, is the complex with $\left(\Sigma^{i} M\right)_{n}=M_{n-i}$ and $\partial_{n}^{\Sigma^{i} M}=(-1)^{i} \partial_{n-i}^{M}$.

The derived category of the category of $R$-modules is denoted $\mathcal{D}(R)$. Isomorphisms in $\mathcal{D}(R)$ are identified by the symbol $\simeq$ and isomorphisms up to shift are designated by $\sim$. 
Fix $R$-complexes $M$ and $N$. Let $\inf (M)$ and $\sup (M)$ denote the infimum and supremum, respectively, of the set $\left\{n \in \mathbb{Z} \mid \mathrm{H}_{n}(M) \neq 0\right\}$, with the conventions $\sup (\emptyset)=-\infty$ and $\inf (\emptyset)=\infty$. The complex $M$ is homologically bounded if $\mathrm{H}_{i}(M)=0$ for all $|i| \gg 0$; it is degree-wise homologically finite if each $\mathrm{H}_{i}(M)$ is finitely generated; and it is homologically finite if $\oplus_{i} \mathrm{H}_{i}(M)$ is finitely generated. If $M$ is degree-wise homologically finite and $\inf (M) \geqslant-\infty$, then $M$ admits a degree-wise finite free resolution, that is, an isomorphism $F \stackrel{\simeq}{\rightarrow} M$ in $\mathcal{D}(R)$ such that each $F_{i}$ is a finitely generated free $R$-module and $F_{i}=0$ for $i<\inf (M)$.

Let $M \otimes_{R}^{\mathbf{L}} N$ and $\mathbf{R} \operatorname{Hom}_{R}(M, N)$ denote the left-derived tensor product and rightderived homomorphism complexes. Let $\operatorname{pd}_{R}(M), \mathrm{fd}_{R}(M)$, and $\operatorname{id}_{R}(M)$ denote the projective, flat, and injective dimensions of $M$, as in 4. A ring homomorphism $R \rightarrow S$ has finite flat dimension if $\operatorname{fd}_{R}(S)$ is finite. When $R$ is a local ring with residue field $k$, the depth of $M$ is $\operatorname{depth}_{R}(M):=-\sup \left(\mathbf{R H o m}_{R}(k, M)\right)$.

The ideas behind semidualizing complexes go back, e.g. to Grothendieck's dualizing complexes 21 and the relative dualizing complexes of Avramov and Foxby [6] 1 The generality that we work in for this paper is from Christensen [11].

Definition 1.2. An $R$-complex $C$ is semidualizing if it is homologically finite and the "homothety morphism" $\chi_{C}^{R}: R \rightarrow \mathbf{R H o m}_{R}(C, C)$ is an isomorphism in $\mathcal{D}(R)$. An $R$-complex $D$ is dualizing if it is semidualizing and has finite injective dimension.

Fact 1.3. If $R$ is a homomorphic image of a Gorenstein ring, e.g., if $R$ is complete, then $R$ admits a dualizing complex by [21, §V.10].

Fact 1.4. Let $\varphi:(R, \mathfrak{m}) \rightarrow(S, \mathfrak{n})$ be a local ring homomorphism of finite flat dimension, and let $M$ be a homologically finite $R$-complex. From [11, (5.7) Proposition] and [17. Theorem 4.5] we know that $S \otimes_{R}^{\mathbf{L}} M$ is semidualizing for $S$ if and only if $M$ is semidualizing for $R$. When $\varphi$ is flat, the complex $S \otimes_{R}^{\mathbf{L}} M$ is dualizing for $S$ if and only if $M$ is dualizing for $R$ and $S / \mathfrak{m} S$ is Gorenstein by [5. (4.2) Proposition, (5.1) Theorem] ${ }^{2}$

The next categories come from Avramov and Foxby [6] and Christensen [11.

Definition 1.5. Let $C$ be a semidualizing $R$-complex. The Auslander class with respect to $C$ is the full subcategory $\mathcal{A}_{C}(R) \subseteq \mathcal{D}(R)$ consisting of the homologically bounded $R$-complexes $M$ such that $C \otimes_{R}^{\mathrm{L}} M$ is homologically bounded and the natural morphism $\gamma_{M}^{C}: M \rightarrow \mathbf{R} \operatorname{Hom}_{R}\left(C, C \otimes_{R}^{\mathbf{L}} M\right)$ is an isomorphism in $\mathcal{D}(R)$. The Bass class with respect to $C$ is the full subcategory $\mathcal{B}_{C}(R) \subseteq \mathcal{D}(R)$ consisting of the homologically bounded $R$-complexes $M$ such that $\mathbf{R H o m} R(C, M)$ is homologically bounded and the natural morphism $\xi_{M}^{C}: C \otimes_{R}^{\mathbf{L}} \mathbf{R} \operatorname{Hom}_{R}(C, M) \rightarrow M$ is an isomorphism in $\mathcal{D}(R)$.

Definition 1.6. Let $C$ and $M$ be $R$-complexes. We set $M^{\dagger} C:=\operatorname{RHom}_{R}(M, C)$ and $M^{\dagger_{C} \dagger^{\dagger}}:=\left(M^{\dagger_{C}}\right)^{\dagger_{C}}$. The $R$-complex $M$ is derived $C$-reflexive when the complexes $M$ and $M^{\dagger_{C}}$ are homologically finite and the "biduality morphism" $\delta_{M}^{C}: M \rightarrow$ $M^{\dagger_{C} \dagger^{\dagger} C}$ is an isomorphism in $\mathcal{D}(R)$. 3

\footnotetext{
${ }^{1}$ The history summarized in this section is skeletal at best. For a more thorough discussion, the interested reader may find 31] helpful.

${ }^{2}$ This can be done more generally using Gorenstein homomorphisms, but we do not need that level of generality here; see [5].

${ }^{3}$ Avramov, Iyengar, and Lipman [10. Theorem 2] show that this definition is redundant when $C$ is semidualizing.
} 
Definition 1.7. Let $C$ be a semidualizing $R$-complex. Set

$$
\mathrm{G}_{C}-\operatorname{dim}_{R}(M):= \begin{cases}\inf (C)-\inf \left(\mathbf{R H o m}_{R}(M, C)\right) & \text { if } M \text { is derived } C \text {-reflexive } \\ \infty & \text { otherwise. }\end{cases}
$$

When $C=R$ we write $\mathrm{G}-\operatorname{dim}_{R}(M)$ in place of $\mathrm{G}_{C}-\operatorname{dim}_{R}(M)$; this is the $G$ dimension of Auslander and Bridger [2] and Yassemi 36].

Fact 1.8 ([11, (3.14) Theorem]). Let $C$ be a semidualizing $R$-complex, and let $M$ be an $R$-complex such that $\mathrm{G}_{C}$ - $\operatorname{dim}_{R}(M)<\infty$. Then

$$
\mathrm{G}_{C}-\operatorname{dim}_{R}(M)=\operatorname{depth}(R)-\operatorname{depth}_{R}(M) .
$$

Fact 1.9. Assume that $R$ has a dualizing complex $D$. Each homologically finite $R$-complex $M$ is derived $D$-reflexive by [21, Proposition V.2.1] or [11, (8.4) Proposition]. Furthermore, for each semidualizing $R$-complex $C$, the complex $C^{\dagger_{D}}$ is also semidualizing; see [11, (2.11) Theorem].

Definition 1.10. Let $R$ be local with residue field $k$, and let $M$ be a homologically finite $R$-complex. The Poincaré and Bass series of $M$ are the formal Laurant series

$$
P_{M}^{R}(t):=\sum_{i \in \mathbb{Z}} \operatorname{dim}_{k}\left(\operatorname{Tor}_{i}^{R}(M, k)\right) t^{i} \quad \text { and } \quad I_{R}^{M}(t):=\sum_{i \in \mathbb{Z}} \operatorname{dim}_{k}\left(\operatorname{Ext}_{R}^{i}(k, M)\right) t^{i} .
$$

Fact 1.11. For a semidualizing $R$-complex $C$ we have from [14, Theorem 4.1(a)]:

$$
I_{R}^{R}(t)=I_{R}^{\mathbf{R H o m}}(C, C)(t)=P_{C}^{R}(t) I_{R}^{C}(t) .
$$

Our next topic is from Avramov, Foxby, and Herzog [7].

Definition 1.12. Let $\varphi:(R, \mathfrak{m}) \rightarrow(S, \mathfrak{n})$ be a local ring homomorphism. The semi-completion of $\varphi$ is the composition $\grave{\varphi}: R \rightarrow \widehat{S}$ of $\varphi$ and the inclusion $S \rightarrow \widehat{S}$.

The map $\varphi$ is said to be weakly regular if it is flat with regular closed fibre. If $\varphi$ is flat, we define the depth and embedding dimension of $\varphi$ to be $\operatorname{depth}(\varphi):=$ $\operatorname{depth}(S / \mathfrak{m} S)$ and $\operatorname{edim}(\varphi):=\operatorname{edim}(S / \mathfrak{m} S)$. If $\varphi$ is weakly regular of embedding dimension 0 , we say that $\varphi$ is weakly étale or that $S$ is a weakly étale $R$-algebra.

A regular (resp. Gorenstein) factorization of $\varphi$ is a diagram of local homomorphisms $R \stackrel{\dot{\varphi}}{\rightarrow} R^{\prime} \stackrel{\varphi^{\prime}}{\longrightarrow} S$ where $\varphi=\varphi^{\prime} \dot{\varphi}, \dot{\varphi}$ is flat, $R^{\prime} / \mathfrak{m} R^{\prime}$ is regular (resp. Gorenstein), and $\varphi^{\prime}$ is surjective. By [7, (1.1) Theorem], the semi-completion $\grave{\varphi}$ admits a regular factorization $R \rightarrow R^{\prime} \rightarrow \widehat{S}$ such that $R^{\prime}$ is complete; this is called a Cohen factorization of $\grave{\varphi}$.

Given a regular factorization $R \stackrel{\dot{\varphi}}{\rightarrow} R^{\prime} \stackrel{\varphi^{\prime}}{\longrightarrow} S$ for $\varphi$, it is straightforward to show that $\operatorname{edim}(\varphi) \geqslant \operatorname{edim}(S / \mathfrak{m} S)$; this factorization is minimal if $\operatorname{edim}(\varphi)=$ $\operatorname{edim}(S / \mathfrak{m} S)$.

The focus of this paper is on $\mathrm{G}_{C}$-dimension of local homomorphisms, though we do require the following slightly greater generality for a few results. See 30].

Definition 1.13. Let $\varphi: R \rightarrow S$ be a local ring homomorphism and $M$ a homologically finite $S$-complex. Fix a semidualizing $R$-complex $C$ and a Cohen factorization $R \stackrel{\dot{\varphi}}{\rightarrow} R^{\prime} \stackrel{\varphi^{\prime}}{\longrightarrow} \widehat{S}$ of the semi-completion $\grave{\varphi}$. The $G_{C}$-dimension of $M$ over $\varphi$ is

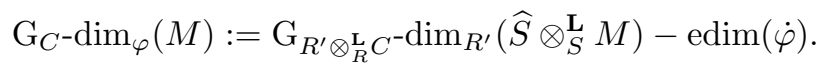

The $G_{C}$-dimension of $\varphi$ is $\mathrm{G}_{C}-\operatorname{dim}(\varphi):=\mathrm{G}_{C}-\operatorname{dim}_{\varphi}(S)$. In the case $C=R$, we follow [23] and set G-dim$\varphi(M):=\mathrm{G}_{R}-\operatorname{dim}_{\varphi}(M)$ and $\mathrm{G}-\operatorname{dim}(\varphi):=\mathrm{G}_{R}$-dim( $(\varphi)$. 
Fact 1.14. Let $\varphi: R \rightarrow S$ be a local homomorphism, $C$ a semidualizing $R$-complex, and $M$ a homologically finite $S$-complex.

(a) The quantities $\mathrm{G}_{C}-\operatorname{dim}_{\varphi}(M), \mathrm{G}_{C}-\operatorname{dim}_{\grave{\varphi}}\left(\widehat{S} \otimes_{S}^{\mathbf{L}} M\right)$, and $\mathrm{G}_{\widehat{R} \otimes_{R} C^{-}}-\operatorname{dim}_{\widehat{\varphi}}\left(\widehat{S} \otimes_{S}^{\mathbf{L}} M\right)$ are simultaneously finite, by an argument as in [23, 3.4.1].

(b) If $\varphi$ admits a Gorenstein factorization $R \stackrel{\dot{\varphi}}{\rightarrow} R^{\prime} \stackrel{\varphi^{\prime}}{\rightarrow} S$, then $\mathrm{G}_{C^{-}}-\operatorname{dim}_{\varphi}(M)=$ $\mathrm{G}_{R^{\prime} \otimes{ }_{R} C^{-}} \operatorname{dim}_{R^{\prime}}(M)-\operatorname{depth}(\dot{\varphi})$, as in [23, 3.8. Proposition].

(c) If $R$ admits a dualizing complex $D$, then $\mathrm{G}_{C}$ - $\operatorname{dim}_{\varphi}(M)<\infty$ if and only if $M$ is in $\mathcal{A}_{C^{\dagger} D}(R)$, by [30, 2.2.3].

Definition 1.15. Let $\varphi: R \rightarrow R$ be a ring endomorphism. For $n=1,2, \ldots$ let $\varphi^{n}$ denote the $n$-fold composition of $\varphi$ with itself. Each endomorphism $\varphi^{n}$ defines a new $R$-module structure on $R$, which we denote as ${ }^{n} R$ : specifically for $r \in R$ and $s \in{ }^{n} R$, we have $r \cdot s=\varphi^{n}(r) s$ If $(R, \mathfrak{m})$ is local, then $\varphi$ is contracting if $\varphi^{n}(\mathfrak{m}) \subseteq \mathfrak{m}^{2}$ for $n \gg 0$.

If $R$ contains a field of characteristic $p>0$, then the Frobenius endomorphism $f_{R}: R \rightarrow R$ given by $r \mapsto r^{p}$ is a contracting endomorphism, and $R$ is $F$-finite when ${ }^{1} R$ is finitely generated over $R$.

\section{Complexes Induced From Ring Homomorphisms}

This section contains foundational results about the following tool from [17] that is central to our study of $\mathrm{G}_{C}$-dimensions of local ring homomorphisms.

Notation 2.1. Let $\varphi: R \rightarrow S$ be a local homomorphism that has a Gorenstein factorization $R \stackrel{\dot{\varphi}}{\rightarrow} R^{\prime} \stackrel{\varphi^{\prime}}{\rightarrow} S$. Given an $R$-complex $M$, we set

$$
M(\varphi):=\Sigma^{d} \mathbf{R} \operatorname{Hom}_{R^{\prime}}\left(S, R^{\prime} \otimes_{R}^{\mathbf{L}} M\right)
$$

where $d=\operatorname{depth}(\dot{\varphi})$.

Remark 2.2. Let $\varphi: R \rightarrow S$ be a local homomorphism that has a Gorenstein factorization, and let $M$ be a homologically finite $R$-complex. The $R$-complex $M(\varphi)$ is independent of the choice of Gorenstein factorization by [17, Theorem 6.5(a)]. If $C$ is semidualizing for $R$, then $C(\varphi)$ is semidualizing for $S$ if and only if $\mathrm{G}_{C}-\operatorname{dim}(\varphi)<\infty$, by Fact 1.4 and [11, (6.1) Theorem]. Also, if $C$ is dualizing for $R$, then $C(\varphi)$ is dualizing for $S$ by [17, Remark 6.7]. If $\varphi$ is module-finite, then $C(\varphi) \simeq \operatorname{RHom}_{R}(S, C)$ by [17, Theorem $\left.6.5(\mathrm{c})\right]$.

Definition 2.3. Let $\varphi: R \rightarrow R$ be a contracting endomorphism. A factorized pushout diagram is a commutative diagram of local ring homomorphisms

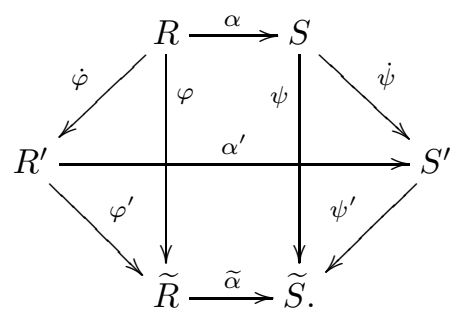

\footnotetext{
${ }^{4}$ This of course depends on $\varphi$, but this notation is fairly standard.
} 
such that the maps $\alpha$ and $\alpha^{\prime}$ have finite flat dimension, the diagrams $R \stackrel{\dot{\varphi}}{\rightarrow} R^{\prime} \stackrel{\varphi^{\prime}}{\longrightarrow} \widetilde{R}$ and $S \stackrel{\dot{\psi}}{\rightarrow} S^{\prime} \stackrel{\psi^{\prime}}{\longrightarrow} \widetilde{S}$ are Gorenstein factorizations of $\varphi$ and $\psi$, respectively, and the natural morphism $S^{\prime} \otimes_{R^{\prime}}^{\mathbf{L}} \widetilde{R} \rightarrow \widetilde{S}$ is an isomorphism.

Remark 2.4. Factorized pushout diagrams exist in at least two important cases:

(1) Consider a commutative diagram of local ring homomorphisms

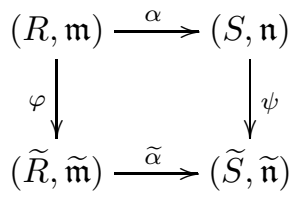

such that $\alpha$ and $\widetilde{\alpha}$ are weakly regular, $\widetilde{S}$ is complete, and the induced map $R / \mathfrak{m} \rightarrow$ $\widetilde{S} / \widetilde{\mathfrak{n}}$ is separable. Assume that $\varphi$ has a minimal regular factorization $R \stackrel{\dot{\varphi}}{\rightarrow} R^{\prime} \stackrel{\varphi^{\prime}}{\longrightarrow} S$, and fix a minimal Cohen factorization $\widetilde{R} \stackrel{\dot{\varphi}}{\rightarrow} S^{\prime} \stackrel{\widetilde{\varphi}^{\prime}}{\rightarrow} \widetilde{S}$ of $\widetilde{\varphi}$. Then [27, Proposition $3.2]$ provides a weakly regular local ring homomorphism $\alpha^{\prime}: R^{\prime} \rightarrow S^{\prime}$ satisfying the hypotheses of Lemma 2.5

(2) For the Frobenius endomorphism, such diagrams are built in Lemma 4.1

The next few results explain behavior of the $M(\varphi)$-construction with respect to factorized pushout diagrams.

Lemma 2.5. Let $C$ be a semidualizing R-complex, and fix a factorized pushout diagram (2.3.1).

(a) The following conditions are equivalent:

(i) $\mathrm{G}_{C}-\operatorname{dim} \varphi<\infty$,

(ii) $\mathrm{G}_{S \otimes_{R}^{L} C}$ - $\operatorname{dim} \psi<\infty$,

(iii) $C(\varphi)$ is semidualizing for $\widetilde{R}$, and

(iv) $\left(S \otimes_{R}^{\mathbf{L}} C\right)(\psi)$ is semidualizing for $\widetilde{S}$.

(b) $\mathrm{G}_{C}-\operatorname{dim} \varphi+\operatorname{edim}(\dot{\varphi})=\mathrm{G}_{S \otimes_{R}^{L} C}-\operatorname{dim} \psi+\operatorname{edim}(\dot{\psi})$.

Proof. From [17, Theorem 4.8] we have

$$
\begin{aligned}
\mathrm{G}_{R^{\prime} \otimes_{R}^{\mathrm{L}} C^{-}} \operatorname{dim}_{R^{\prime}}(\widetilde{R}) & =\mathrm{G}_{S^{\prime} \otimes_{R^{\prime}}^{\mathrm{L}}\left(R^{\prime} \otimes_{R}^{\mathrm{L} C}\right)^{-}} \operatorname{dim}_{S^{\prime}}\left(S^{\prime} \otimes_{R^{\prime}} \widetilde{R}\right) \\
& =\mathrm{G}_{S^{\prime} \otimes_{S}^{\mathrm{L}}\left(S \otimes_{R}^{\mathrm{L}} C\right)^{-}}-\operatorname{dim}_{S^{\prime}}(\widetilde{S}) .
\end{aligned}
$$

This explains part (b) and the equivalence (i) $\Longleftrightarrow$ (ii) from part (国). Since $S \otimes_{R}^{\mathbf{L}} C$ is semidualizing for $S$ by Fact 1.4 the equivalences (i) $\Longleftrightarrow$ (iii) and (ii) $\Longleftrightarrow$ (iv) from part (国) are by Remark 2.2

Lemma 2.6. Consider a factorized pushout diagram (2.3.1). Given a homologically finite $R$-complex $M$, there is an isomorphism $\left(S \otimes_{R}^{\mathbf{L}} M\right)(\psi) \simeq \Sigma^{d} \widetilde{S} \otimes_{\widetilde{R}}^{\mathbf{L}} M(\varphi)$ in $\mathcal{D}(S)$ where $d=\operatorname{depth}(\dot{\psi})-\operatorname{depth}(\dot{\varphi})$. 
Proof. In the following sequence of isomorphisms in $\mathcal{D}(S)$, the first and fourth steps are by definition, and the second one is from the assumptions on diagram (2.3.1):

$$
\begin{aligned}
\left(S \otimes_{R}^{\mathbf{L}} M\right)(\psi) & \simeq \Sigma^{\operatorname{depth}\left(S^{\prime} / \mathfrak{n} S^{\prime}\right)} \mathbf{R H o m}_{S^{\prime}}\left(\widetilde{S}, S^{\prime} \otimes_{S}^{\mathbf{L}}\left(S \otimes_{R}^{\mathbf{L}} M\right)\right) \\
& \simeq \Sigma^{\operatorname{depth}\left(S^{\prime} / \mathfrak{n} S^{\prime}\right)} \mathbf{R} \operatorname{Hom}_{S^{\prime}}\left(S^{\prime} \otimes_{R^{\prime}}^{\mathbf{L}} \widetilde{R}, S^{\prime} \otimes_{R^{\prime}}^{\mathbf{L}}\left(R^{\prime} \otimes_{R}^{\mathbf{L}} M\right)\right) \\
& \simeq \Sigma^{\operatorname{depth}\left(S^{\prime} / \mathfrak{n} S^{\prime}\right)} S^{\prime} \otimes_{R^{\prime}}^{\mathbf{L}} \mathbf{R} \operatorname{Hom}_{R^{\prime}}\left(\widetilde{R}, R^{\prime} \otimes_{R}^{\mathbf{L}} M\right) \\
& \simeq \Sigma^{d} S^{\prime} \otimes_{R^{\prime}}^{\mathbf{L}} M(\varphi) \\
& \simeq \Sigma^{d}\left(S^{\prime} \otimes_{R^{\prime}}^{\mathbf{L}} \widetilde{R}\right) \otimes_{\widetilde{R}}^{\mathbf{L}} M(\varphi) \\
& \simeq \Sigma^{d} \widetilde{S} \otimes_{\widetilde{R}}^{\mathbf{L}} M(\varphi) .
\end{aligned}
$$

The third isomorphism follows from [4, 4.4 Lemma], and the others are routine.

Lemma 2.7. Consider a factorized pushout diagram (2.3.1) such that $\widetilde{R}=R$ and $\widetilde{S}=S$ and $\widetilde{\alpha}=\alpha$, that is, such that $\varphi$ and $\psi$ are endomorphisms. Let $C$ be a semidualizing $R$-complex. Then $C \sim C(\varphi)$ as $R$-complexes if and only if $S \otimes_{R}^{\mathbf{L}} C \sim\left(S \otimes_{R}^{\mathbf{L}} C\right)(\psi)$ as $S$-complexes.

Proof. Since $C$ is semidualizing over $R$ and $\operatorname{fd}(\alpha)<\infty$, we know that $S \otimes_{R}^{\mathbf{L}} C$ is semidualizing for $S$ by Fact 1.4 .

For the forward implication, assume that $C \sim C(\varphi)$ as $R$-complexes. This implies

$$
S \otimes_{R}^{\mathbf{L}} C \sim S \otimes_{R}^{\mathbf{L}}(C(\varphi)) \sim\left(S \otimes_{R}^{\mathbf{L}} C\right)(\psi)
$$

by Lemma 2.6.

For the converse, assume that $S \otimes_{R}^{\mathbf{L}} C \sim\left(S \otimes_{R}^{\mathbf{L}} C\right)(\psi)$ as $S$-complexes. In particular, this implies that $\left(S \otimes_{R}^{\mathbf{L}} C\right)(\psi)$ is semidualizing for $S$, so Lemma 2.5(a) implies that $C(\varphi)$ is semidualizing over $R$. Now, since

$$
S \otimes_{R}^{\mathbf{L}} C \sim\left(S \otimes_{R}^{\mathbf{L}} C\right)(\psi) \sim S \otimes_{R}^{\mathbf{L}}(C(\varphi))
$$

we conclude $C \sim C(\varphi)$ by [17, Theorem 4.9].

Lemma 2.8. Fix a factorized pushout diagram (2.3.1) such that $\widetilde{R}=R$ and $\widetilde{S}=S$ and $\widetilde{\alpha}=\alpha$, that is, such that $\varphi$ and $\psi$ are endomorphisms. Let $C$ be a semidualizing $R$-complex. Assume that $\mathrm{G}_{C}-\operatorname{dim}(\varphi)<\infty$. Then $C$ is derived $C(\varphi)$-reflexive over $R$ if and only if $S \otimes_{R}^{\mathbf{L}} C$ is derived $\left(S \otimes_{R}^{\mathbf{L}} C\right)(\psi)$-reflexive over $S$.

Proof. Lemma 2.5(a) implies that $C(\varphi)$ is semidualizing for $R$, and $\left(S \otimes_{R}^{\mathbf{L}} C\right)(\psi)$ is semidualizing for $S$. Thus, the equivalence follows from Lemma 2.6 and [17, Theorem 4.8] as in the proof of Lemma 2.7.

The next two results document behavior of the $M(\varphi)$-construction with respect to semi-completions and compositions.

Lemma 2.9. Let $R \stackrel{\varphi}{\rightarrow} S$ be a local homomorphism that admits a Gorenstein factorization, and consider the semi-completion $R \stackrel{\grave{\varphi}}{\rightarrow} \widehat{S}$. Given a homologically finite $R$-complex $M$, there is an isomorphism $M(\grave{\varphi}) \simeq \widehat{S} \otimes_{S}^{\mathbf{L}} M(\varphi)$ in $\mathcal{D}(\widehat{S})$. 
Proof. Let $R \stackrel{\dot{\varphi}}{\rightarrow} R^{\prime} \stackrel{\varphi^{\prime}}{\rightarrow} S$ be a Gorenstein factorization of $\varphi$. Consider the following commutative diagram where the new maps are the natural ones:

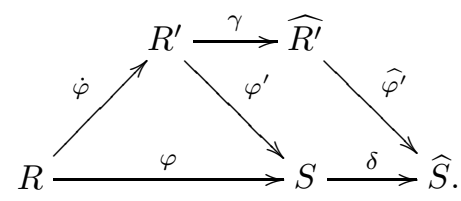

Since $\varphi^{\prime}$ is surjective, we have $\widehat{S} \simeq \widehat{R^{\prime}} \otimes \mathbb{R}^{\prime}, S$, and the diagram $R \stackrel{\gamma \dot{\varphi}}{\longrightarrow} \widehat{R^{\prime}} \stackrel{\widehat{\varphi^{\prime}}}{\longrightarrow} \widehat{S}$ is a Gorenstein factorization of $\grave{\varphi}=\delta \varphi$. Also, we have $d:=\operatorname{depth}(\dot{\varphi})=\operatorname{depth}(\gamma \dot{\varphi})$. These explain the first, second, and last steps in the next sequence:

$$
\begin{aligned}
M(\grave{\varphi}) & \simeq \Sigma^{d} \mathbf{R} \operatorname{Hom}_{\widehat{R^{\prime}}}\left(\widehat{S}, \widehat{R^{\prime}} \otimes_{R}^{\mathbf{L}} M\right) \\
& \simeq \Sigma^{d} \mathbf{R} \operatorname{Hom}_{\widehat{R^{\prime}}}\left(\widehat{R^{\prime}} \otimes_{R^{\prime}}^{\mathbf{L}} S, \widehat{R^{\prime}} \otimes_{R^{\prime}}^{\mathbf{L}}\left(R^{\prime} \otimes_{R}^{\mathbf{L}} M\right)\right) \\
& \simeq \Sigma^{d} \widehat{R^{\prime}} \otimes_{R^{\prime}}^{\mathbf{L}} \mathbf{R H o m}_{R^{\prime}}\left(S, R^{\prime} \otimes_{R}^{\mathbf{L}} M\right) \\
& \simeq \widehat{R^{\prime}} \otimes_{R^{\prime}}^{\mathbf{L}} \Sigma^{d} \mathbf{R} \operatorname{Hom}_{R^{\prime}}\left(S, R^{\prime} \otimes_{R}^{\mathbf{L}} M\right) \\
& \simeq\left(\widehat{R^{\prime}} \otimes_{R^{\prime}}^{\mathbf{L}} S\right) \otimes_{S}^{\mathbf{L}} \Sigma^{d} \mathbf{R} \operatorname{Hom}_{R^{\prime}}\left(S, R^{\prime} \otimes_{R}^{\mathbf{L}} M\right) \\
& \simeq \widehat{S} \otimes_{S}^{\mathbf{L}} M(\varphi) .
\end{aligned}
$$

The third step is by flat base change since $S$ is finite over $R^{\prime}$. The remaining steps are routine.

Lemma 2.10. Let $(R, \mathfrak{m}) \stackrel{\varphi}{\rightarrow}(S, \mathfrak{n}) \stackrel{\psi}{\rightarrow} T$ be local homomorphisms such that $\varphi, \psi$, and $\psi \varphi$ admit Gorenstein factorizations. Given a homologically finite $R$-complex $M$, there is an isomorphism $M(\psi \varphi) \simeq M(\varphi)(\psi)$ in $\mathcal{D}(T)$.

Proof. Case 1: $T$ is complete. Let $R \stackrel{\dot{\varphi}}{\rightarrow} R^{\prime} \stackrel{\varphi^{\prime}}{\longrightarrow} S$ be a Gorenstein factorization of $\varphi$, and let $S \stackrel{\dot{\psi}}{\rightarrow} S^{\prime} \stackrel{\psi^{\prime}}{\rightarrow} T$ be a Cohen factorization of $\psi$. Then $S^{\prime}$ is complete, so the map $R^{\prime} \stackrel{\dot{\psi} \varphi^{\prime}}{\longrightarrow} S^{\prime}$ has a minimal Cohen factorization $R^{\prime} \stackrel{\dot{\rho}}{\rightarrow} R^{\prime \prime} \stackrel{\rho^{\prime}}{\rightarrow} S^{\prime}$. Note that it follows from the proof of [7. (1.6) Theorem] that $S^{\prime} \simeq R^{\prime \prime} \otimes_{R^{\prime}}^{\mathbf{L}} S$. From this we conclude that $\dot{\psi}$ and $\dot{\rho}$ have isomorphic closed fibres. In particular, we have $\operatorname{depth}(\dot{\rho})=\operatorname{depth}(\dot{\psi})$.

Set $d^{\prime}=\operatorname{depth}(\dot{\psi})$ and $d^{\prime \prime}=\operatorname{depth}(\dot{\varphi})$. We claim that the composition $\dot{\rho} \dot{\varphi}$ is Gorenstein and flat, and that

$$
d:=\operatorname{depth}(\dot{\rho} \dot{\varphi})=\operatorname{depth}(\dot{\rho})+\operatorname{depth}(\dot{\varphi})=\operatorname{depth}(\dot{\psi})+\operatorname{depth}(\dot{\varphi})=d^{\prime}+d^{\prime \prime} .
$$

Indeed, the composition of flat local homomorphisms is flat and local. Furthermore, the induced map $R^{\prime} / \mathfrak{m} R^{\prime} \stackrel{\bar{\rho}}{\rightarrow} R^{\prime \prime} / \mathfrak{m} R^{\prime \prime}$ is flat and local with closed fibre $R^{\prime \prime} / \mathfrak{m}^{\prime} R^{\prime \prime}$ where $\mathfrak{m}^{\prime}$ is the maximal ideal of $R^{\prime}$. Since $R^{\prime} / \mathfrak{m} R^{\prime}$ and $R^{\prime \prime} / \mathfrak{m}^{\prime} R^{\prime \prime}$ are both Gorenstein by assumption, the fact that $\overline{\dot{\rho}}$ is flat and local implies that $R^{\prime \prime} / \mathfrak{m} R^{\prime \prime}$ is Gorenstein, so $\dot{\rho} \dot{\varphi}$ is Gorenstein. Furthermore, the fact that $\overline{\dot{\rho}}$ is flat and local explains 
the second equality in the next sequence

$$
\begin{aligned}
\operatorname{depth}(\dot{\rho} \dot{\varphi}) & =\operatorname{depth}\left(R^{\prime \prime} / \mathfrak{m} R^{\prime \prime}\right) \\
& =\operatorname{depth}\left(R^{\prime} / \mathfrak{m} R^{\prime}\right)+\operatorname{depth}\left(R^{\prime \prime} / \mathfrak{m}^{\prime} R^{\prime \prime}\right) \\
& =\operatorname{depth}(\dot{\rho})+\operatorname{depth}(\dot{\varphi}) \\
& =\operatorname{depth}(\dot{\psi})+\operatorname{depth}(\dot{\varphi}) .
\end{aligned}
$$

The last step follows from the fact that $\dot{\rho}$ and $\dot{\psi}$ are both flat and have isomorphic closed fibres. This establishes the claim.

Thus, the diagram $R \stackrel{\dot{\rho} \dot{\varphi}}{\longrightarrow} R^{\prime \prime} \stackrel{\psi^{\prime} \rho^{\prime}}{\longrightarrow} T$ is a Gorenstein factorization of $\psi \varphi$ :

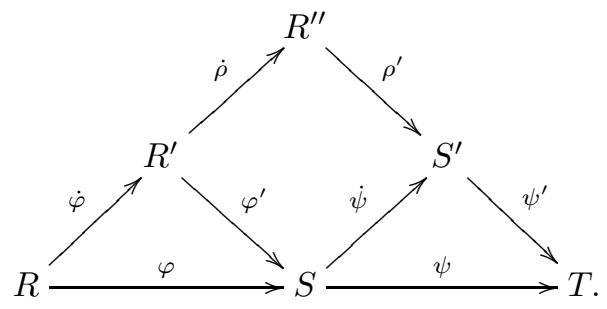

This explains the first, third, sixth, and eighth steps in the next display:

$$
\begin{aligned}
M(\varphi)(\psi) & =\Sigma^{d^{\prime \prime}} \mathbf{R H o m}_{S^{\prime}}\left(T, S^{\prime} \otimes_{S}^{\mathbf{L}} \Sigma^{d^{\prime}} \mathbf{R H o m}_{R^{\prime}}\left(S, R^{\prime} \otimes_{R}^{\mathbf{L}} M\right)\right) \\
& \simeq \Sigma^{d^{\prime}+d^{\prime \prime}} \mathbf{R H o m}_{S^{\prime}}\left(T, S^{\prime} \otimes_{S}^{\mathbf{L}} \mathbf{R H o m}_{R^{\prime}}\left(S, R^{\prime} \otimes_{R}^{\mathbf{L}} M\right)\right) \\
& \simeq \Sigma^{d} \mathbf{R H o m}_{S^{\prime}}\left(T,\left(R^{\prime \prime} \otimes_{R^{\prime}}^{\mathbf{L}} S\right) \otimes_{S}^{\mathbf{L}} \mathbf{R H o m}_{R^{\prime}}\left(S, R^{\prime} \otimes_{R}^{\mathbf{L}} M\right)\right) \\
& \simeq \Sigma^{d} \mathbf{R H o m}_{S^{\prime}}\left(T, R^{\prime \prime} \otimes_{R^{\prime}}^{\mathbf{L}} \mathbf{R} \operatorname{Hom}_{R^{\prime}}\left(S, R^{\prime} \otimes_{R}^{\mathbf{L}} M\right)\right) \\
& \simeq \Sigma^{d} \mathbf{R H o m}_{S^{\prime}}\left(T, \mathbf{R H o m}_{R^{\prime \prime}}\left(R^{\prime \prime} \otimes_{R^{\prime}}^{\mathbf{L}} S, R^{\prime \prime} \otimes_{R^{\prime}}^{\mathbf{L}}\left(R^{\prime} \otimes_{R}^{\mathbf{L}} M\right)\right)\right) \\
& \simeq \Sigma^{d} \mathbf{R} \operatorname{Hom}_{S^{\prime}}\left(T, \mathbf{R H o m} R^{\prime \prime}\left(S^{\prime}, R^{\prime \prime} \otimes_{R}^{\mathbf{L}} M\right)\right) \\
& \simeq \Sigma^{d} \mathbf{R} \operatorname{Hom}_{R^{\prime \prime}}\left(T, R^{\prime \prime} \otimes_{R}^{\mathbf{L}} M\right) \\
& =M(\psi \varphi) .
\end{aligned}
$$

The seventh step is Hom-tensor adjointness, and the others are routine.

Case 2: the general case. Let $\grave{\psi}: S \rightarrow \widehat{T}$ be the semi-completion of $\psi$. Note that $\grave{\psi} \varphi: R \rightarrow \widehat{T}$ is the semi-completion of $\psi \varphi$. Thus, Lemma 2.9 explains the first and third isomorphisms in the next sequence

$$
\widehat{T} \otimes_{T}^{\mathbf{L}} M(\psi \varphi) \simeq M(\grave{\psi} \varphi) \simeq M(\varphi)(\grave{\psi}) \simeq \widehat{T} \otimes_{T}^{\mathbf{L}}(M(\varphi)(\psi))
$$

and the second isomorphism is from Case 1 since $\widehat{T}$ is complete. Hence, the conclusion $M(\psi \varphi) \simeq M(\varphi)(\psi)$ follows from [17, Lemma 1.10].

The interested reader may want to compare our next two results to [17, Proposition 6.10] which assumes that $\operatorname{fd}(\varphi)$ is finite.

Proposition 2.11. Let $R \stackrel{\varphi}{\rightarrow} S$ be a local homomorphism that admits a Gorenstein factorization, and let $C$ be a semidualizing $R$-complex.

(a) Then one has $I_{S}^{C(\varphi)}(t)=I_{R}^{C}(t)$.

(b) If $\mathrm{G}_{C}-\operatorname{dim}(\varphi)$ is finite, then $P_{C(\varphi)}^{S}(t)=P_{C}^{R}(t) I_{S}^{S}(t) / I_{R}^{R}(t)$. 
Proof. (国) In the following display, the first equality is by definition:

$$
\begin{aligned}
I_{S}^{C(\varphi)}(t) & =I_{S}^{\sum^{d} \mathbf{R H o m}_{R^{\prime}}\left(S, R^{\prime} \otimes_{R}^{\mathrm{L}} C\right)}(t) \\
& =t^{-d} I_{S}^{\mathbf{R} \operatorname{Hom}_{R^{\prime}}\left(S, R^{\prime} \otimes_{R}^{\mathrm{L}} C\right)}(t) \\
& =t^{-d} I_{R^{\prime}}^{R^{\prime} \mathbb{L}_{R} C}(t) \\
& =t^{-d} I_{R^{\prime}}^{R^{\prime}}(t) / P_{R^{\prime} \otimes_{R} R^{\prime}}^{R^{\prime}}(t) \\
& =t^{-d} I_{R}^{R}(t) I_{R^{\prime} / \mathfrak{m} R^{\prime}}^{R^{\prime}}(t) / P_{C}^{R}(t) \\
& =t^{-d} I_{R}^{R}(t) t^{d} / P_{C}^{R}(t) \\
& =I_{R}^{R}(t) / P_{C}^{R}(t) \\
& =I_{R}^{C}(t) .
\end{aligned}
$$

The third equality is from [11, (1.7.8) Lemma]. The fourth and eighth equalities are by Fact 1.11. The fifth equality is from [16, Theorem]. The sixth equality is from the fact that $\dot{\varphi}$ is Gorenstein of depth $d$, and the remaining equalities are routine.

(b) Assume that $\mathrm{G}_{C}-\operatorname{dim}(\varphi)$ is finite, that is, that $C(\varphi)$ is a semidualizing $S$ complex; see Remark 2.2. Thus, Fact 1.11 explains the first and third equalities in the next display:

$$
\begin{aligned}
I_{R}^{R}(t) P_{C(\varphi)}^{S}(t) & =I_{R}^{R}(t) I_{S}^{S}(t) / I_{S}^{C(\varphi)}(t) \\
& =I_{R}^{R}(t) I_{S}^{S}(t) / I_{R}^{C}(t) \\
& =I_{R}^{R}(t) I_{S}^{S}(t) /\left[I_{R}^{R}(t) / P_{C}^{R}(t)\right] \\
& =P_{C}^{R}(t) I_{S}^{S}(t) .
\end{aligned}
$$

The second equality is from part (国), and the fourth equality is routine.

Corollary 2.12. Let $R \stackrel{\varphi}{\rightarrow} R$ be a local endomorphism. Assume that $n$ is a positive integer such that $\varphi^{n}$ admits a Gorenstein factorization, and let $C$ be a semidualizing $R$-complex.

(a) Then one has $I_{R}^{C\left(\varphi^{n}\right)}(t)=I_{R}^{C}(t)$.

(b) If $\mathrm{G}_{C}-\operatorname{dim}\left(\varphi^{n}\right)$ is finite, then $P_{C\left(\varphi^{n}\right)}^{R}(t)=P_{C}^{R}(t)$.

Proof. This follows directly from Proposition 2.11 since $S=R$ in this case.

\section{Results about Contracting Endomorphisms}

This section contains the proof of Theorem $\mathrm{A}$ from the introduction and other similar results for arbitrary contracting endomorphisms. We begin with a version of [23, 7.3. Corollary] for our situation.

Proposition 3.1. Let $\varphi:(R, \mathfrak{m}) \rightarrow S$ be a local homomorphism and $M$ a complex of $S$-modules that is homologically finite over $R$. Let $C$ be a semidualizing $R$-complex. Then $\mathrm{G}_{C}$ - $\operatorname{dim}_{\varphi}(M)=\mathrm{G}_{C}$-dim $\operatorname{dim}_{R}(M)$. In particular, the quantities $\mathrm{G}_{C}$-dim$\varphi(M)$ and $\mathrm{G}_{C}-\operatorname{dim}_{R}(M)$ are simultaneously finite.

Proof. Let $\widetilde{S}$ be the m-adic completion of $S$, and let $\widetilde{\varphi}: \widehat{R} \rightarrow \widetilde{S}$ be the induced map. Let $\widehat{\varphi}: \widehat{R} \rightarrow \widehat{S}$ denote the map induced on completions, and set $\widehat{C}=\widehat{R} \otimes_{R}^{\mathbf{L}} C$. Consider the Koszul complex $K=K^{R}(\mathfrak{m})$ on a minimal generating sequence for $\mathfrak{m}$. 
Arguing as in the proof of [23, 5.1. Theorem], and using [17, Theorem 4.4], we can replace $M$ with $K \otimes_{R}^{\mathbf{L}} M$ to assume that $\mathfrak{m}$ annihilates the homology of $M$. (The results [30, Proposition 4.1(a)] and [13, Proposition 4.1(i)] may be helpful here.) As in the proof of [23, 7.1. Theorem], it follows that $\widetilde{S} \otimes_{S}^{\mathbf{L}} M \simeq \widehat{R} \otimes_{R}^{\mathbf{L}} M$ in $\mathcal{D}(\widehat{R})$.

Since the completion of $\widetilde{\varphi}$ at the maximal ideal of $\widetilde{S}$ is $\widehat{\varphi}$, Fact 1.14 implies that $\mathrm{G}_{C^{-}} \operatorname{dim}_{\varphi}(M)<\infty$ if and only if $\mathrm{G}_{\widehat{C}^{-}} \operatorname{dim}_{\widetilde{\varphi}}\left(\widetilde{S} \otimes_{S}^{\mathbf{L}} M\right)<\infty$ if and only if $\widetilde{S} \otimes_{S}^{\mathbf{L}} M \in$ $\mathcal{A}_{\widehat{C}^{\dagger} D}(\widehat{R})$, where $D$ is a dualizing complex for $\widehat{R}$. Using the isomorphism $\widetilde{S} \otimes_{S}^{\mathbf{L}} M \simeq$ $\widehat{R} \otimes{ }_{R}^{\mathbf{L}} M$, we conclude that $\mathrm{G}_{C^{-}} \operatorname{dim}_{\varphi}(M)<\infty$ if and only if $\widehat{R} \otimes_{R}^{\mathbf{L}} M \in \mathcal{A}_{\widehat{C}^{\dagger} D}(\widehat{R})$, that is, if and only if $\mathrm{G}_{\widehat{C}^{-}}-\operatorname{dim}_{\widehat{R}}\left(\widehat{R} \otimes_{R}^{\mathbf{L}} M\right)<\infty$, by [11, (4.7) Theorem]. Because of the equality $\mathrm{G}_{\widehat{C}^{-}} \operatorname{dim}_{\widehat{R}}\left(\widehat{R} \otimes_{R}^{\mathrm{L}} M\right)=\mathrm{G}_{C}$ - $\operatorname{dim}_{R}(M)$ from [11, (5.11) Corollary], it follows that $\mathrm{G}_{C}$ - $\operatorname{dim}_{\varphi}(M)<\infty$ if and only if $\mathrm{G}_{C}$ - $\operatorname{dim}_{R}(M)<\infty$.

For the rest of the proof, assume that $\mathrm{G}_{C}-\operatorname{dim}_{\varphi}(M)$ and $\mathrm{G}_{C}-\operatorname{dim}_{R}(M)$ are finite. As in the proof of [23, 3.5. Theorem], using Fact [1.8, we have the first equality in the following display:

$$
\begin{aligned}
\mathrm{G}_{C}-\operatorname{dim}_{\varphi}(M) & =\operatorname{depth}(R)-\operatorname{depth}_{S}(M) \\
& =\operatorname{depth}(R)-\operatorname{depth}_{R}(M) \\
& =\mathrm{G}_{C}-\operatorname{dim}_{R}(M) .
\end{aligned}
$$

The other equalities are from [23, 2.8. Lemma] and Fact 1.8,

The next result implies Theorem $\mathrm{A}$ from the introduction; see 3.4

Theorem 3.2. Let $\varphi: R \rightarrow R$ be a contracting endomorphism, and let $C$ be a semidualizing R-complex. Assume that $\varphi^{n}$ has a Gorenstein factorization for each $n \geqslant 1$, e.g., this holds when $\varphi$ is module-finite or $R$ is complete. Then the following are equivalent:

(i) $C$ is a dualizing $R$-complex.

(ii) $C \sim C\left(\varphi^{n}\right)$ for some $n>0$.

(iii) $\mathrm{G}_{C}-\operatorname{dim} \varphi^{n}<\infty$ and $C$ is derived $C\left(\varphi^{n}\right)$-reflexive for some $n>0$.

(iv) $\mathrm{G}_{C}$-dim $\varphi^{n}<\infty$ for infinitely many $n>0$.

If $R$ has a dualizing complex $D$, then these conditions are equivalent to the following:

(v) $\mathrm{G}_{C}-\operatorname{dim} \varphi^{n}<\infty$ and ${ }^{n} R \otimes_{R}^{L} C^{\dagger D}$ is derived $C^{\dagger_{D}}$-reflexive for some $n>0$.

Proof. (ii) $\Longrightarrow$ (iii) Assume that $C$ is a dualizing $R$-complex. By Remark 2.2 the complex $C\left(\varphi^{n}\right)$ is dualizing for $R$. Since dualizing complexes are unique up to shift in $\mathcal{D}(R)$, we have $C \sim C\left(\varphi^{n}\right)$.

(iii) $\Longrightarrow$ (iii) Assume that $C \sim C\left(\varphi^{n}\right)$ for some $n>0$. Since $C$ is semidualizing $R$-complex, the condition $C \sim C\left(\varphi^{n}\right)$ implies that $C\left(\varphi^{n}\right)$ is semidualizing $R$ complex. Remark 2.2 implies that $\mathrm{G}_{C^{-}} \operatorname{dim} \varphi^{n}<\infty$. Since $C$ is derived $C$-reflexive, the condition $C \sim C\left(\varphi^{n}\right)$ implies that $C$ is derived $C\left(\varphi^{n}\right)$-reflexive.

(iii) $\Longrightarrow$ (iv) Assume that $\mathrm{G}_{C^{-}} \operatorname{dim} \varphi^{n}<\infty$ and $C$ is derived $C\left(\varphi^{n}\right)$-reflexive for some $n>0$. Remark 2.2 implies that $C\left(\varphi^{n}\right)$ is semidualizing, and Corollary 2.12(b) implies that $C\left(\varphi^{n}\right)$ has the same Poincaré series as $C$. Thus, we have $C\left(\varphi^{n}\right) \sim C$ by the proof of [31, Fact 2.28].

Thus, Lemma 2.10 implies that

$$
C\left(\varphi^{2 n}\right) \simeq\left(C\left(\varphi^{n}\right)\right)\left(\varphi^{n}\right) \sim C\left(\varphi^{n}\right) \sim C .
$$


Thus, we have $\mathrm{G}_{C}$ - $\operatorname{dim}_{R}\left(\varphi^{2 n}\right)<\infty$ by Remark 2.2. Inductively, one shows that $\mathrm{G}_{C}$ - $\operatorname{dim}_{R}\left(\varphi^{m n}\right)<\infty$ for all $m \in \mathbb{N}$, hence condition (iv) follows.

(iv) $\Longrightarrow$ (ii) Assume that $\mathrm{G}_{C}$ - $\operatorname{dim} \varphi^{n}<\infty$ for infinitely many $n>0$. Fact 1.14 implies that $\mathrm{G}_{C}$ - $\operatorname{dim} \varphi^{n}<\infty$ if and only if $\mathrm{G}_{\widehat{R} \otimes_{R}^{\mathrm{L} C}}$ - $\operatorname{dim} \widehat{\varphi}^{n}<\infty$. Also, we know that $C$ is dualizing for $R$ if and only if $\widehat{R} \otimes_{R}^{\mathbf{L}} C$ is dualizing for $\widehat{R}$. Furthermore, $\widehat{\varphi}^{n}$ has a Cohen factorization for each $n$ since $\widehat{R}$ is complete. Thus, by passing to $\widehat{R}$, one may assume that $R$ is complete. Hence, $R$ has a dualizing complex $D$ by Fact [1.3. Note that by [11, (2.12) Corollary], the $R$-complex $C^{\dagger_{D}}$ is semidualizing.

By our hypothesis, $\mathrm{G}_{C}$-dim $\varphi^{n}<\infty$ for infinitely many $n$. Thus by Fact 1.14((C)), we have ${ }^{n} R \in \mathcal{A}_{C^{\dagger} D}(R)$ and hence ${ }^{n} R \otimes_{R}^{\mathrm{L}} C^{\dagger_{D}}$ is homologically bounded for infinitely many $n$. Therefore, for infinitely many $n$ we have $\operatorname{Tor}_{i}^{R}\left({ }^{n} R, C^{\dagger} D\right)=0$ for all $i \gg$ 0. Now [23, 6.4. Proposition] implies $\operatorname{pd}_{R}\left(C^{\dagger} D\right)<\infty$ and this is equivalent to $\operatorname{id}_{R}(C)<\infty$. Thus $C$ is a dualizing complex for $R$.

To complete the proof, we assume that $R$ has a dualizing complex $D$ and prove (iii) $\Longleftrightarrow(\mathrm{v})$. To this end, we assume that $n$ is a positive integer such that $\mathrm{G}_{C}$-dim $\varphi^{n}<\infty$, and we prove that $C$ is derived $C\left(\varphi^{n}\right)$-reflexive if and only if ${ }^{n} R \otimes_{R}^{\mathbf{L}} C^{\dagger_{D}}$ is derived $C^{\dagger_{D}}$-reflexive. Let $R \rightarrow R^{\prime} \rightarrow R$ be a Gorenstein factorization of $\varphi^{n}$.

We use the following fact from [17, Proposition 3.9]: If $A$ and $B$ are semidualizing $R$-complexes, then $A$ is derived $B$-reflexive if and only if $B^{\dagger_{D}}$ is derived $A^{\dagger^{-} D_{-}}$ reflexive. Thus, to complete the proof, we need only show that $\left({ }^{n} R \otimes_{R}^{\mathbf{L}} C^{\dagger^{\dagger} D}\right)^{\dagger^{\dagger} D} \sim$ $C\left(\varphi^{n}\right)$. To this end, the first step in the next sequence is from Remark 2.2 .

$$
\begin{aligned}
\left({ }^{n} R \otimes_{R}^{\mathbf{L}} C^{\dagger D}\right)^{\dagger D} & \sim \mathbf{R H o m}_{n_{R}}\left({ }^{n} R \otimes_{R}^{\mathbf{L}} C^{\dagger D}, D\left(\varphi^{n}\right)\right) \\
& \simeq \mathbf{R H o m}_{R}\left(C^{\dagger_{D}}, D\left(\varphi^{n}\right)\right) \\
& \sim \mathbf{R H o m}_{R}\left(C^{\dagger_{D}}, \mathbf{R} \operatorname{Hom}_{R^{\prime}}\left({ }^{n} R, R^{\prime} \otimes_{R}^{\mathbf{L}} D\right)\right) \\
& \simeq \mathbf{R H o m}_{R^{\prime}}\left({ }^{n} R \otimes_{R}^{\mathbf{L}} C^{\dagger D}, R^{\prime} \otimes_{R}^{\mathbf{L}} D\right) \\
& \simeq \mathbf{R H o m}_{R^{\prime}}\left({ }^{n} R \otimes_{R^{\prime}}^{\mathbf{L}}\left(R^{\prime} \otimes_{R}^{\mathbf{L}} C^{\dagger_{D}}\right), R^{\prime} \otimes_{R}^{\mathbf{L}} D\right) \\
& \simeq \mathbf{R H o m}_{R^{\prime}}\left({ }^{n} R, \mathbf{R} \operatorname{Hom}_{R^{\prime}}\left(R^{\prime} \otimes_{R}^{\mathbf{L}} C^{\dagger_{D}}, R^{\prime} \otimes_{R}^{\mathbf{L}} D\right)\right) \\
& \simeq \mathbf{R H o m}_{R^{\prime}}\left({ }^{n} R, R^{\prime} \otimes_{R}^{\mathbf{L}} \mathbf{R} \operatorname{Hom}_{R}\left(C^{\dagger D}, D\right)\right) \\
& \simeq \mathbf{R H o m}_{R^{\prime}}\left({ }^{n} R, R^{\prime} \otimes_{R}^{\mathbf{L}} C\right) \\
& \sim C\left(\varphi^{n}\right) .
\end{aligned}
$$

The second, fourth, and sixth steps are from Hom-tensor adjointness. The seventh step is by flat base change. The eighth step is by Fact 1.9, and the other steps are routine.

Remark 3.3. In Theorem 3.2 (and its successors) we have more equivalent conditions, but they become tedious to write down. For instance, the given conditions are equivalent to the following:

(iii) $C \sim C\left(\varphi^{n}\right)$ for all $n>0$.

Indeed, this condition clearly implies condition (iii) from Theorem 3.2 And the proof of Theorem 3.2 shows that (ii) $\Longrightarrow$ (ii). One verifies similarly that the other conditions in Theorem 3.2 can be replaced with "for all" versions as well.

3.4 (Proof of Thoerem A). Use Proposition 3.1, Remark 2.2 and Theorem 3.2. 
To state and prove results that allow us, for instance, to pass to the completion, we introduce and briefly study the following class of diagrams.

Definition 3.5. Let $\varphi: R \rightarrow R$ be a contracting endomorphism. A commutative diagram of local ring homomorphisms

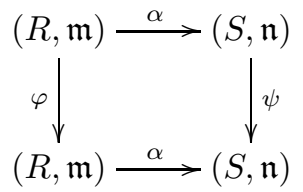

is cows if $S$ is complete, the map $\alpha$ is weakly regular, and the map $R / \mathfrak{m} \rightarrow S / \mathfrak{n}$ induced by $\alpha \varphi$ is separable.

Remark 3.6. Let $\varphi: R \rightarrow R$ be a contracting endomorphism. One always has a trivial cows diagram (3.5.1): use the natural map $\alpha: R \rightarrow \widehat{R}$ and $\psi=\widehat{\varphi}$. More interestingly, Proposition A.3 shows that if the $R / \varphi(\mathfrak{m}) R$ is artinian and induced map $\bar{\varphi}: k \rightarrow k$ is separable, then there is a cows diagram (3.5.1) such that $\psi$ is module-finite. (See also Lemma A.2) Thus, conditions (ii') and (iii') in Theorem 3.8 say that questions about $G_{C}$-dimensions (in the separable case) can be reduced to the module-finite case, like reducing a Frobenius question to the $F$-finite case; c.f. Theorem 4.2 .

From another perspective, one reason to study cows diagrams is found in their similarity to Cohen factorizations: when the map $\psi$ is module-finite, it detects properties of $\varphi$ like the surjective part $\varphi^{\prime}$ of a Cohen factorization for $\hat{\varphi}$ or $\grave{\varphi}$. To see what we mean by this, recall that one point of considering $\varphi^{\prime}$ is given by the fact that many homological properties of $\varphi$ can be detected by $\varphi^{\prime}$. For instance, the map $\varphi$ is quasi-Gorenstein if and only if $\varphi^{\prime}$ is quasi-Gorenstein. We have seen similarly that many homological properties of $\varphi$ can be detected by $\psi$ : e.g., under certain hypotheses, $\varphi$ is quasi-Gorenstein if and only if $\psi$ is quasi-Gorenstein; see [27, Theorem B].

Lemma 3.7. Every cows diagram (3.5.1) gives rise to a commutative diagram

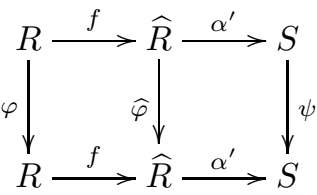

of local ring homomorphisms such that the second square is cows and $\alpha=\alpha^{\prime} f$ where $f: R \rightarrow \widehat{R}$ is the natural map. Conversely, given a cows diagram for $\widehat{\varphi}$ as in the second square of (3.7.1), the following diagram is cows:

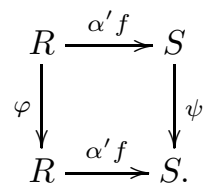

Proof. Given a commutative diagram (3.5.1), since $S$ is complete the local homomorphism $\alpha$ factors through $\widehat{R}$, so there is a local homomorphism $\alpha^{\prime}$ making (3.7.1) commute. Conversely, given a commutative diagram as in the second 
square of (3.7.1), since the first square of (3.7.1) commutes, it follows that the diagram (3.7.2) also commutes. Thus, it remains to show that the second square of (3.7.1) is cows if and only if 3.7.2 is cows.

By construction the induced maps $R / \mathfrak{m} \rightarrow S / \mathfrak{n}$ and $\widehat{R} / \mathfrak{m} \widehat{R} \rightarrow S / \mathfrak{n}$ are the same, so one is separable if and only the other is separable. Thus, it remains to show that $\alpha^{\prime}$ is weakly regular if and only if $\alpha^{\prime} f$ is weakly regular. Since $f$ is weakly regular and the composition of weakly regular maps is weakly regular, one implication is routine. For the converse, assume that $\alpha^{\prime} f$ is weakly regular. Since $\alpha^{\prime}$ and $\alpha^{\prime} f$ have the same closed fibres, it suffices to show that $\alpha^{\prime}$ is flat. This follows from the sequence $\operatorname{Tor}_{i}^{R}(R / \mathfrak{m}, S) \cong \operatorname{Tor}_{i}^{\widehat{R}}(\widehat{R} / \mathfrak{m} \widehat{R}, S)=0$ for $i \geqslant 1$; see [1, Lemme II.57] or [4, 5.5 Proposition $(\mathrm{F})]$.

Theorem 3.8. Let $\varphi: R \rightarrow R$ be a contracting endomorphism, and let $C$ be a semidualizing $R$-complex. Then the following conditions are equivalent:

(i) $C$ is a dualizing $R$-complex.

(i') There is a cows diagram (3.5.1) such that $S \otimes_{R}^{\mathbf{L}} C$ is dualizing for $S$.

(ii) $\widehat{R} \otimes_{R}^{\mathbf{L}} C \sim\left(\widehat{R} \otimes_{R}^{\mathbf{L}} C\right)\left(\widehat{\varphi}^{n}\right)$ for some $n>0$.

(ii') There is a cows diagram (3.5.1) such that $S \otimes_{R}^{\mathbf{L}} C \sim\left(S \otimes_{R}^{\mathbf{L}} C\right)\left(\psi^{n}\right)$ for some $n>0$.

(iii) $\mathrm{G}_{C}$-dim $\varphi^{n}<\infty$ and $\widehat{R} \otimes_{R}^{\mathbf{L}} C$ is derived $\left(\widehat{R} \otimes_{R}^{\mathbf{L}} C\right)\left(\widehat{\varphi}^{n}\right)$-reflexive for some $n>0$.

(iii') There is a cows diagram 3.5.1 such that $\mathrm{G}_{S \otimes_{R} C^{-}}-\operatorname{dim} \psi^{n}<\infty$ and $S \otimes{ }_{R}^{\mathbf{L}} C$ is derived $\left(S \otimes_{R}^{\mathbf{L}} C\right)\left(\psi^{n}\right)$-reflexive for some $n>0$.

(iv) $\mathrm{G}_{C}$-dim $\varphi^{n}<\infty$ for infinitely many $n>0$.

(iv') There is a cows diagram (3.5.1) such that $\mathrm{G}_{S \otimes_{R}^{\mathrm{L} C}}$-dim $\psi^{n}<\infty$ for infinitely many $n>0$.

(v') There is a cows diagram (3.5.1) such that $\mathrm{G}_{S \otimes_{R} C^{-}}$dim $\psi^{n}<\infty$ and such that ${ }^{n} S \otimes_{S}^{\mathbf{L}} \mathbf{R} \operatorname{Hom}_{S}\left(S \otimes{ }_{R}^{\mathbf{L}} C, D^{S}\right)$ is derived $\mathbf{R} \operatorname{Hom}_{S}\left(S \otimes_{R}^{\mathbf{L}} C, D^{S}\right)$-reflexive for some $n>0$, where $D^{S}$ is a dualizing $S$-complex.

If $R$ has a dualizing complex $D$, then these conditions are equivalent to the following:

(v) $\mathrm{G}_{C}$-dim $\varphi^{n}<\infty$ and ${ }^{n} R \otimes_{R}^{L} C^{\dagger_{D}}$ is derived $C^{\dagger^{D}}$-reflexive for some $n>0$.

Proof. The equivalences (i) $\Longleftrightarrow$ (i') and (iv) $\Longleftrightarrow$ (iv') are from Fact 1.4 and Lemma 2.5(a).

For the rest of the proof, we consider two cases.

Case 1: $R$ is complete. In this case, Theorem 3.2 shows that we need only prove the equivalences (ii) $\Longleftrightarrow$ (ii'), (iii) $\Longleftrightarrow$ (iii'), and (v) $\Longleftrightarrow\left(\mathrm{v}^{\prime}\right)$. Consider a cows diagram (3.5.1). Remark 2.4 (1) provides a a factorized pushout diagram (2.3.1) such that $\widetilde{R}=R$ and $\widetilde{S}=S$ and $\widetilde{\alpha}=\alpha$. The equivalence (ii) $\Longleftrightarrow$ (ii') now follows from Lemma 2.7, and (iii) $\Longleftrightarrow$ (iii') follows from Lemmas 2.5(a) and 2.8.

For the equivalence $(\mathrm{v}) \Longleftrightarrow\left(\mathrm{v}^{\prime}\right)$ in this case, since $R$ is complete, it has a dualizing complex $D$. Using Lemma 2.5(或) again, we see that $\mathrm{G}_{S \otimes{ }_{R}} C^{-\operatorname{dim}} \psi^{n}<\infty$ if and only if $\mathrm{G}_{C}$-dim $\varphi^{n}<\infty$. Assume for the remainder of this paragraph that $\mathrm{G}_{S \otimes \mathbb{R}^{\mathrm{L}} C^{-}} \operatorname{dim} \psi^{n}<\infty$. Since $\alpha$ is flat, there are isomorphisms in $\mathcal{D}(S)$

$$
\begin{gathered}
\mathbf{R H o m}_{S}\left(S \otimes_{R}^{\mathbf{L}} C, S \otimes_{R}^{\mathbf{L}} D\right) \simeq S \otimes_{R}^{\mathbf{L}} C^{\dagger^{D}} \\
{ }^{n} S \otimes_{S}^{\mathbf{L}} \mathbf{R H o m}_{S}\left(S \otimes_{R}^{\mathbf{L}} C, S \otimes_{R}^{\mathbf{L}} D\right) \simeq S \otimes_{R}^{\mathbf{L}}\left({ }^{n} R \otimes_{R}^{\mathbf{L}} C^{\dagger D}\right) .
\end{gathered}
$$


Thus, ${ }^{n} S \otimes_{S}^{\mathbf{L}} \mathbf{R} \operatorname{Hom}_{S}\left(S \otimes_{R}^{\mathbf{L}} C, S \otimes_{R}^{\mathbf{L}} D\right)$ is derived $\mathbf{R} \operatorname{Hom}_{S}\left(S \otimes_{R}^{\mathbf{L}} C, S \otimes_{R}^{\mathbf{L}} D\right)$-reflexive if and only if ${ }^{n} R \otimes_{R}^{\mathbf{L}} C^{\dagger D}$ is derived $C^{\dagger D}$-reflexive, by [11, (5.10) Theorem].

Case 2: the general case. Fact 1.4 shows that (i) is equivalent to

(1) $\widehat{R} \otimes_{R}^{\mathbf{L}} C$ is a dualizing $\widehat{R}$-complex.

From Fact 1.14 we see that conditions (iii) and (iv) are equivalent (respectively) to the following:

(3) $\mathrm{G}_{\widehat{R} \otimes \mathrm{L}_{R} C^{-}} \operatorname{dim} \widehat{\varphi}^{n}<\infty$ and $\widehat{R} \otimes_{R}^{\mathbf{L}} C$ is derived $\left(\widehat{R} \otimes_{R}^{\mathbf{L}} C\right)\left(\widehat{\varphi}^{n}\right)$-reflexive for some $n>0$.

(4) $\mathrm{G}_{\widehat{R} \otimes_{R} C^{-}}$dim $\widehat{\varphi}^{n}<\infty$ for infinitely many $n>0$.

Claim: Condition (ii') is equivalent to the following:

(2') There is a cows diagram

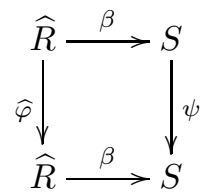

such that $S \otimes_{\widehat{R}}^{\mathbf{L}}\left(\widehat{R} \otimes_{R}^{\mathbf{L}} C\right) \sim\left(S \otimes_{\widehat{R}}^{\mathbf{L}}\left(\widehat{R} \otimes_{R}^{\mathbf{L}} C\right)\right)\left(\psi^{n}\right)$ for some $n>0$.

In light of Lemma 3.7, this follows from the isomorphisms

$$
\left(S \otimes_{\widehat{R}}^{\mathbf{L}}\left(\widehat{R} \otimes_{R}^{\mathbf{L}} C\right)\right)\left(\psi^{n}\right) \simeq\left(S \otimes_{R}^{\mathbf{L}} C\right)\left(\psi^{n}\right) \quad S \otimes_{\widehat{R}}^{\mathbf{L}}\left(\widehat{R} \otimes_{R}^{\mathbf{L}} C\right) \simeq S \otimes_{R}^{\mathbf{L}} C .
$$

Similar reasoning shows that conditions (iii') and ( $\left.\mathrm{v}^{\prime}\right)$ are equivalent (respectively) to the following:

(3') There is a cows diagram (3.8.1) such that $\mathrm{G}_{S \otimes_{R}^{\mathbf{L}} C^{-}} \operatorname{dim} \psi^{n}<\infty$ and $S \otimes \widehat{L}_{R}\left(\widehat{R} \otimes_{R}^{\mathbf{L}}\right.$ $C)$ is derived $\left(S \otimes{ }_{\widehat{R}}^{\mathbf{L}}\left(\widehat{R} \otimes_{R}^{\mathbf{L}} C\right)\right)\left(\psi^{n}\right)$-reflexive for some $n>0$.

(5') There is a cows diagram (3.8.1) such that $\mathrm{G}_{S \otimes_{R} C^{\mathrm{L}}}$-dim $\psi^{n}<\infty$ and such that ${ }^{n} S \otimes_{S}^{\mathbf{L}} \mathbf{R} \operatorname{Hom}_{S}\left(S \otimes \widehat{\mathbf{L}}_{\widehat{R}}\left(\widehat{R} \otimes_{R}^{\mathbf{L}} C\right), D^{S}\right)$ is derived $\mathbf{R} \operatorname{Hom}_{S}\left(S \otimes \otimes_{\widehat{R}}^{\mathbf{L}}\left(\widehat{R} \otimes{ }_{R}^{\mathbf{L}} C\right), D^{S}\right)$ reflexive for some $n>0$, where $D^{S}$ is a dualizing $S$-complex.

Claim: if $R$ has a dualizing complex, then condition (v) is equivalent to:

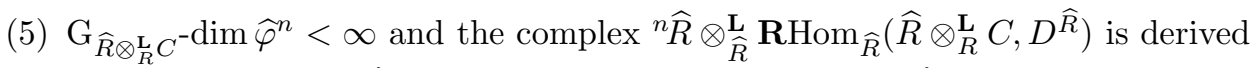

$\mathbf{R H o m}_{\widehat{R}}\left(\widehat{R} \otimes \mathbb{L}_{R} C, D^{\widehat{R}}\right)$-reflexive for some $n>0$ where $D^{\widehat{R}}$ is dualizing for $\widehat{R}$.

Fact 1.14 implies that $\mathrm{G}_{\widehat{R} \otimes C_{R} C^{-}}-\operatorname{dim} \widehat{\varphi}^{n}<\infty$ if and only if $\mathrm{G}_{C^{-}} \operatorname{dim} \varphi^{n}<\infty$. From Fact 1.4 we know that $\widehat{R} \otimes_{R}^{\mathrm{L}} D$ is dualizing for $\widehat{R}$, so we have $\widehat{R} \otimes_{R}^{\mathrm{L}} D \sim D^{\widehat{R}}$. The complex $\widehat{R} \otimes_{R}^{\mathbf{L}} C$ is semidualizing for $\widehat{R}$, hence so is

$$
\mathbf{R H o m}_{\widehat{R}}\left(\widehat{R} \otimes_{R}^{\mathbf{L}} C, D^{\widehat{R}}\right) \sim \mathbf{R H o m}_{\widehat{R}}\left(\widehat{R} \otimes_{R}^{\mathbf{L}} C, \widehat{R} \otimes_{R}^{\mathbf{L}} D\right) \simeq \widehat{R} \otimes_{R}^{\mathbf{L}} C^{\dagger D} .
$$

In $\mathcal{D}(\widehat{R})$ we have

$$
{ }^{n} \widehat{R} \otimes_{\widehat{R}}^{\mathbf{L}} \mathbf{R H o m}_{\widehat{R}}\left(\widehat{R} \otimes_{R}^{\mathbf{L}} C, D^{\widehat{R}}\right) \sim{ }^{n} \widehat{R} \otimes_{\widehat{R}}^{\mathbf{L}}\left(\widehat{R} \otimes_{R}^{\mathbf{L}} C^{\dagger D}\right) \sim \widehat{R} \otimes_{R}^{\mathbf{L}}\left({ }^{n} R \otimes_{R}^{\mathbf{L}} C^{\dagger D}\right)
$$

Thus, the $\widehat{R}$-complex ${ }^{n} \widehat{R} \otimes \widehat{\mathbb{R}}_{\widehat{L}}^{\mathbf{L}} \mathbf{R} \operatorname{Hom}_{\widehat{R}}\left(\widehat{R} \otimes_{R}^{\mathbf{L}} C, D^{\widehat{R}}\right)$ is derived $\mathbf{R H o m}_{\widehat{R}}\left(\widehat{R} \otimes_{R}^{\mathbf{L}} C, D^{\widehat{R}}\right)$ reflexive if and only if $\widehat{R} \otimes_{R}^{\mathbf{L}}\left({ }^{n} R \otimes_{R}^{\mathbf{L}} C^{\dagger_{D}}\right)$ is derived $\widehat{R} \otimes_{R}^{\mathbf{L}} C^{\dagger_{D}}$-reflexive; by [11, (5.10) Theorem], this second condition occurs if and only if ${ }^{n} R \otimes_{R}^{\mathbf{L}} C^{\dagger_{D}}$ is derived $C^{\dagger D}$-reflexive. This completes the proof of the claim. 
By Case 1, conditions (1), (2), (2'), (3), (3'), (4), (5') and (5) are equivalent. Thus, the corresponding conditions (i), (ii), etc. are equivalent.

Remark 3.9. As in Remark 3.3, we note here that in Theorem 3.8 (and subsequent results) we have more equivalent conditions. For instance, the given conditions are equivalent to the following:

$\left(\overline{i i^{\prime}}\right)$ For every cows diagram (3.5.1), we have $S \otimes_{R}^{\mathbf{L}} C \sim\left(S \otimes_{R}^{\mathbf{L}} C\right)\left(\psi^{n}\right)$ for all $n>0$.

Next, we consider versions of Theorems 3.2 and 3.8 using Bass class conditions. A tool for this is the following generalization of [28, Theorem A] for complexes.

Lemma 3.10. Let $R \rightarrow S$ be a local ring homomorphism, and let $M$ be a homologically finite $S$-complex. Assume that $\varphi: R \rightarrow R$ is a contracting endomorphism. Assume that there are infinitely many $n \in \mathbb{N}$ such that there is an integer $t_{n}>\sup (M)$ such that $\operatorname{Ext}_{R}^{i}\left(M,{ }^{n} R\right)=0$ for $t_{n} \leqslant i \leqslant t_{n}+\operatorname{depth}(R)$. Then $\operatorname{pd}_{R}(M)<\infty$.

Proof. Set $\operatorname{depth}(R)=d$, and let $F$ be a degree-wise finite $S$-free resolution of $M$. Set $j=\sup (M)$ and $M^{\prime}=\operatorname{Coker}\left(\partial_{j+1}^{F}\right)$. Then the complex

$$
\cdots \rightarrow F_{j+1} \rightarrow F_{j} \rightarrow M^{\prime} \rightarrow 0
$$

is a degree-wise finite $S$-free resolution of $M^{\prime}$. It follows that for $i \geqslant j+1$ we have

$$
\operatorname{Ext}_{R}^{i-j}\left(M^{\prime},{ }^{n} R\right) \cong \operatorname{Ext}_{R}^{i}\left(M,{ }^{n} R\right) .
$$

From our Ext-vanishing assumption, there are infinitely many $n \in \mathbb{N}$ such that there is an integer $t_{n}^{\prime}=t_{n}-j>0$ such that $\operatorname{Ext}_{R}^{i}\left(M^{\prime},{ }^{n} R\right)=0$ for $t_{n}^{\prime} \leqslant i \leqslant t_{n}^{\prime}+d$. By the proof of [28, Theorem A], we conclude that $\operatorname{pd}_{R}\left(M^{\prime}\right)<\infty$, and it follows that $\operatorname{pd}_{R}(M)<\infty$.

Theorem 3.11. Let $\varphi: R \rightarrow R$ be a contracting endomorphism, and let $C$ be a semidualizing $R$-complex. Then $C \sim R$ in $\mathcal{D}(R)$ if and only if ${ }^{n} R \in \mathcal{B}_{C}(R)$ for infinitely many $n \geqslant 1$.

Proof. The forward implication is straightforward since $\mathcal{B}_{R}(R)$ contains all $R$ modules. For the converse, assume that ${ }^{n} R \in \mathcal{B}_{C}(R)$ for infinitely many $n \geqslant 1$. In particular, there are infinitely many $n \in \mathbb{N}$ such that $\mathbf{R H o m}_{R}\left(C,{ }^{n} R\right)$ is homologically bounded. Hence, there are infinitely many $n \in \mathbb{N}$ such that there is an integer $t_{n}>\sup (C)$ such that $\operatorname{Ext}_{R}^{i}\left(C,{ }^{n} R\right)=0$ for $t_{n} \leqslant i \leqslant t_{n}+\operatorname{depth}(R)$. Lemma 3.10 implies that $\operatorname{pd}_{R}(C)<\infty$, so $C \sim R$ by [11, (8.1) Theorem].

The next three lemmas are for use in the Bass class version of Theorem 3.8, see Theorem 3.15 below.

Lemma 3.12. Let $R \stackrel{\varphi}{\rightarrow} R_{1}$ be a ring homomorphism, and let $C$ be a semidualizing $R$-complex. Let $L$ and $N$ be $R_{1}$-complexes such that $\mathrm{fd}_{R_{1}}(L)<\infty$. If $N \in \mathcal{B}_{C}(R)$, then $L \otimes \mathbb{R}_{1} N \in \mathcal{B}_{C}(R)$; the converse holds when $L$ is a faithfully flat $R_{1}$-module.

Proof. Since $L$ has finite flat dimension over $R_{1}$, tensor evaluation [4, 4.4 Lemma] provides the isomorphism $\mathbf{R H o m}_{R}(C, N) \otimes_{R_{1}}^{\mathbf{L}} L \stackrel{\simeq}{\longrightarrow} \mathbf{R H o m}_{R}\left(C, N \otimes_{R_{1}}^{\mathbf{L}} L\right)$. Thus, if $\mathbf{R H o m}_{R}(C, N)$ is homologically bounded, then so is $\mathbf{R H o m}_{R}\left(C, N \otimes_{R_{1}}^{\mathbf{L}} L\right)$; and the converse holds when $L$ is a faithfully flat $R_{1}$-module. 
Next, consider the commutative diagram wherein the upper horizontal isomorphism is from the previous paragraph:

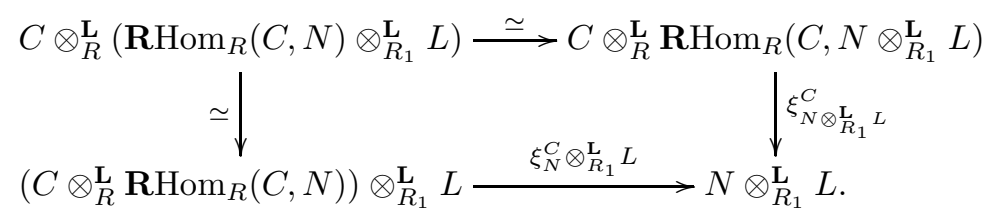

From this, we conclude that $\xi_{N}^{C} \otimes_{R_{1}}^{\mathbf{L}} L$ is an isomorphism if and only if $\xi_{N \otimes_{R_{1}}{ }^{\mathbf{L}}{ }}$ is an isomorphism. Thus, if $\xi_{N}^{C}$ is an isomorphism (hence $\xi_{N}^{C} \otimes_{R_{1}}^{\mathrm{L}} L$ is an isomorphism), then so is $\xi_{N \otimes_{R_{1}}^{\mathrm{L}} L}^{C}$. When $L$ is a faithfully flat $R_{1}$-module and $\xi_{N \otimes_{R_{1}}^{\mathrm{L}} L}^{C}$ is an isomorphism, then $\xi_{N}^{C} \otimes_{R_{1}}^{\mathbf{L}} L$ is an isomorphism, so faithful flatness implies that $\xi_{N}^{C}$ is an isomorphism.

Lemma 3.13. Let $R \stackrel{\varphi}{\rightarrow} R_{1} \stackrel{\alpha}{\rightarrow} S$ be ring homomorphisms, and let $C$ be a semidualizing $R$-complex. Assume that $\alpha$ is flat. If $R_{1} \in \mathcal{B}_{C}(R)$, then $S \in \mathcal{B}_{C}(R)$; the converse holds when $\alpha$ is faithfully flat, e.g., when $\alpha$ is local.

Proof. Use $N=R_{1}$ and $L=S$ in Lemma 3.12 .

Lemma 3.14. For every cows diagram (3.5.1) and every $n \in \mathbb{N}$, one has ${ }^{n} R \in$ $\mathcal{B}_{C}(R)$ if and only if ${ }^{n} S \in \mathcal{B}_{S \otimes_{R}^{\mathrm{L}} C}(S)$.

Proof. The cows diagram (3.5.1) yields a commutative diagram

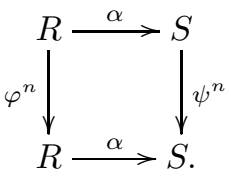

Since $\alpha$ is faithfully flat, Lemma 3.13 shows that ${ }^{n} R \in \mathcal{B}_{C}(R)$ if and only if ${ }^{n} S \in$ $\mathcal{B}_{C}(R)$, and [11, (5.3.a) Proposition] shows that ${ }^{n} S \in \mathcal{B}_{C}(R)$ if and only if ${ }^{n} S \in$ $\mathcal{B}_{S \otimes_{R}^{\mathrm{L}} C}(S)$.

Theorem 3.15. Let $\varphi: R \rightarrow R$ be a contracting endomorphism, and let $C$ be a semidualizing $R$-complex. Then the following conditions are equivalent:

(i) $R$ is Gorenstein.

(ii) $\mathrm{G}_{C}$ - $\operatorname{dim} \varphi^{m}<\infty$ for all $m>0$, and ${ }^{n} R \in \mathcal{B}_{C}(R)$ for all $n>0$.

(ii') For every cows diagram (3.5.1), one has $\mathrm{G}_{S \otimes_{R} C^{-}}-\operatorname{dim} \psi^{m}<\infty$ for all $m>0$, and ${ }^{n} S \in \mathcal{B}_{S \otimes_{R}^{\mathrm{L}} C}(S)$ for all $n>0$.

(iii) $\mathrm{G}_{C}-\operatorname{dim} \varphi^{m}<\infty$ for infinitely many $m>0$, and ${ }^{n} R \in \mathcal{B}_{C}(R)$ for some $n>0$.

(iii') There is a cows diagram (3.5.1) such that $\mathrm{G}_{S \otimes_{R}^{\mathrm{L} C}}-\operatorname{dim} \psi^{m}<\infty$ for infinitely many $m>0$, and ${ }^{n} S \in \mathcal{B}_{S \otimes_{R}^{\mathrm{L}} C}(S)$ for some $n>0$.

(iv) $\mathrm{G}_{C}$ - $\operatorname{dim} \varphi^{m}<\infty$ for some $m>0$, and ${ }^{n} R \in \mathcal{B}_{C}(R)$ for infinitely many $n>0$.

(iv') There is a cows diagram (3.5.1) such that $\mathrm{G}_{S \otimes_{R}^{\mathrm{L}} C^{-}}$dim $\psi^{m}<\infty$ for some $m>0$, and ${ }^{n} S \in \mathcal{B}_{S \otimes_{R}^{\mathrm{L}} C}(S)$ for infinitely many $n>0$.

Proof. The implications (ii) $\Longrightarrow$ (iii) and (ii) $\Longrightarrow$ (iv) are trivial. The equivalences (ii) $\Longleftrightarrow$ (ii'), (iii) $\Longleftrightarrow$ (iii'), and (iv) $\Longleftrightarrow$ (iv') follow from Lemma 3.14

(i) $\Longrightarrow$ (ii) Assume that $R$ is Gorenstein. Then we know from [11, (8.6) Corollary] that $C \sim R$, so $\mathcal{B}_{C}(R)=\mathcal{B}_{R}(R)$ contains every $R$-module, in particular ${ }^{n} R \in \mathcal{B}_{C}(R)$ 
for all $n>0$. Also, since $R$ is Gorenstein, we have $\mathrm{G}_{C^{-}} \operatorname{dim} \varphi^{m}=\mathrm{G}-\operatorname{dim} \varphi^{m}<\infty$ for all $m>0$ by [23, 6.6. Theorem].

(iii) $\Longrightarrow$ (i) Assume that $\mathrm{G}_{C}$-dim $\varphi^{m}<\infty$ for infinitely many $m>0$, and ${ }^{n} R \in \mathcal{B}_{C}(R)$ for some $n>0$. Theorem 3.8 implies that $C$ is dualizing for $R$.

Case 1: $R$ is complete. In this case, $\varphi^{n}$ has a Cohen factorization $R \stackrel{\dot{\tau}}{\rightarrow} R^{\prime} \stackrel{\tau^{\prime}}{\rightarrow}$ $R$. From [11, (5.3.b) Proposition] the condition ${ }^{n} R \in \mathcal{B}_{C}(R)$ implies that $R \in$ $\mathcal{B}_{R^{\prime} \otimes_{R}^{\mathrm{L} C}}\left(R^{\prime}\right)$. As $C$ is dualizing for $R$ and $\dot{\tau}$ is weakly regular, it follows that $R^{\prime} \otimes_{R}^{\mathrm{L}} C$ is dualizing for $R^{\prime}$. Because of [12, 4.4. Theorem], we conclude that $\operatorname{G}^{-} \operatorname{id}_{R^{\prime}}(R)<\infty 5$ In particular, the local ring $R^{\prime}$ has a cyclic module of finite G-injective dimension, so $R^{\prime}$ is Gorenstein by [15, Theorem A]. The fact that $\dot{\tau}$ is flat and local implies that $R$ is Gorenstein.

Case 2: the general case. The ring $R$ is Gorenstein if and only if $\widehat{R}$ is Gorenstein. Since $\mathrm{G}_{C}$-dim $\varphi^{m}<\infty$ for infinitely many $m>0$, Fact 1.14 implies that $\mathrm{G}_{\widehat{R} \otimes{ }_{R} C^{-}}$-dim $\widehat{\varphi}^{m}<\infty$ for infinitely many $m>0$. By Case 1, it suffices to show that the assumption ${ }^{n} R \in \mathcal{B}_{C}(R)$ implies that ${ }^{n} \widehat{R} \in \mathcal{B}_{\widehat{R} \otimes_{R}^{\mathrm{L} C}}(\widehat{R})$. Consider the commutative diagram of local ring homomorphisms

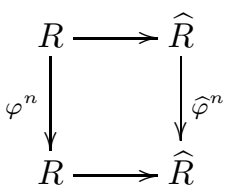

where the unspecified maps are the natural ones. The assumption ${ }^{n} R \in \mathcal{B}_{C}(R)$ implies that ${ }^{n} \widehat{R} \in \mathcal{B}_{C}(R)$ by Lemma 3.13. From [11, (5.3.b) Proposition] we conclude that ${ }^{n} \widehat{R} \in \mathcal{B}_{\widehat{R} \otimes_{R} \mathrm{~L}_{C}}(\widehat{R})$, as desired.

(iv) $\Longrightarrow$ (i) Assume that $\mathrm{G}_{C}$-dim $\varphi^{m}<\infty$ for some $m>0$, and ${ }^{n} R \in \mathcal{B}_{C}(R)$ for infinitely many $n>0$. Theorem 3.11 implies that $C \sim R$ in $\mathcal{D}(R)$. Thus, the assumption $\mathrm{G}_{C}$-dim $\varphi^{m}<\infty$ translates to G-dim $\varphi^{m}<\infty$, and we conclude from [23, 6.6. Theorem] that $R$ is Gorenstein.

\section{Results Specific to the Frobenius Endomorphism}

We begin this section with a combination of [9, Proposition 12.2.7] and [20, Proposition (0.10.3.1)].

Lemma 4.1. Let $(R, \mathfrak{m}, k)$ be a local ring of prime characteristic $p>0$, and let $k \subseteq K$ be a field extension. Then there is a commutative diagram of local ring homomorphisms

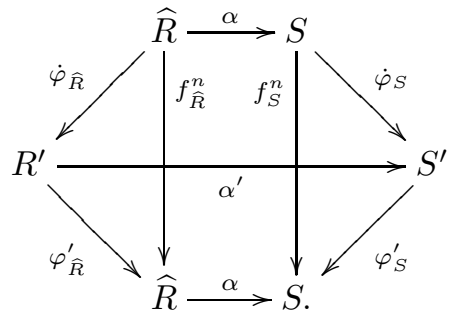

${ }^{5}$ See 12,15 for background on G-injective dimension. 
such that the maps $\alpha$ and $\alpha^{\prime}$ are weakly étale, the rings $S$ and $S^{\prime}$ are complete, the diagrams $\widehat{R} \stackrel{\dot{\varphi}_{\widehat{R}}}{\longrightarrow} R^{\prime} \stackrel{\varphi_{\widehat{R}}^{\prime}}{\longrightarrow} \widehat{R}$ and $S \stackrel{\dot{\varphi}_{S}}{\longrightarrow} S^{\prime} \stackrel{\varphi_{S}^{\prime}}{\longrightarrow} S$ are minimal Cohen factorizations of $f_{\widehat{R}}^{n}$ and $f_{S}^{n}$, respectively, the natural map $S^{\prime} \otimes_{R^{\prime}}^{\mathbf{L}} \widehat{R} \rightarrow S$ is an isomorphism, and the induced map $R / \mathfrak{m} \rightarrow S / \mathfrak{m} S$ is the given field extension $k \subseteq K$. (In particular, this is a factorized pushout diagram.) If $K$ is $F$-finite, then so are $S$ and $S^{\prime}$.

Proof. This conclusion is unchanged if we replace $R$ by $\widehat{R}$, so we assume that $R$ is complete. By Cohen's Structure Theorem there exist integers $e, m \geqslant 0$ and elements $f_{1}, \cdots, f_{m} \in k \llbracket x_{1}, \ldots, x_{e} \rrbracket$ such that $R \cong k \llbracket x_{1}, \ldots, x_{e} \rrbracket /\left(f_{1}, \ldots, f_{m}\right)$, and the images of $x_{1}, \ldots, x_{e}$ in $R$ minimally generate $\mathfrak{m}$. Set $\mathbf{x}=x_{1}, \ldots, x_{e}$, and use the notation $\mathbf{x}^{\mathbf{a}}=x_{1}^{a_{1}} \cdots x_{e}^{a_{e}}$ for all $\mathbf{a}=\left(a_{1}, \ldots, a_{e}\right) \in \mathbb{N}^{e}$. Also, set $\mathbf{f}=f_{1}, \cdots, f_{m}$. We identify $R$ with the ring $k \llbracket \mathbf{x} \rrbracket /(\mathbf{f})$ for the remainder of the proof.

Let $S=K \llbracket \mathbf{x} \rrbracket /(\mathbf{f})$, and let $\alpha: R \rightarrow S$ be induced by the inclusion $k \subseteq K$. Then $S$ is a complete local ring, and $\alpha$ makes $S$ into a local $R$-algebra of characteristic $p$. It is straightforward to show that the map $k \llbracket \mathbf{x} \rrbracket \rightarrow K \llbracket \mathbf{x} \rrbracket$ induced by the inclusion $k \subseteq K$ is flat (e.g., using [26, Exercise 22.3]). Hence, $\alpha$ is flat by base-change. Moreover, $\alpha$ is weakly étale since the maximal ideal of $S$ is $(\mathbf{x}) S=\mathfrak{m} S$ by construction.

We use the following notation of [9, Proposition 12.2.7]. For $g=\sum_{\mathbf{a} \in \mathbb{N}^{e}} r_{\mathbf{a}} \mathbf{x}^{\mathbf{a}}$, set $g^{\left[p^{n}\right]}=\sum_{\mathbf{a} \in \mathbb{N}^{e}} r_{\mathbf{a}}^{p^{n}} \mathbf{x}^{\mathbf{a}}$. Then a minimal Cohen factorization of $f_{R}^{n}$ is given by the following maps. The weakly regular part is $\dot{\varphi}_{R}: k \llbracket \mathbf{x} \rrbracket /(\mathbf{f}) \rightarrow k \llbracket \mathbf{x} \rrbracket /\left(\mathbf{f}^{\left[p^{n}\right]}\right) \llbracket \mathbf{y} \rrbracket$ given by $\dot{\varphi}_{R}(g+(\mathbf{f})):=g^{\left[p^{n}\right]}+\left(\mathbf{f}^{\left[p^{n}\right]}\right)$ where $\mathbf{y}=y_{1}, \ldots, y_{e}$ is another list of variables. The surjective part is the composition $\varphi_{R}^{\prime}=\phi_{R}^{\prime \prime} \rho_{R}$ where $\phi_{R}^{\prime \prime}$ and $\rho_{R}$ are defined next. First, we have $\rho_{R}: k \llbracket \mathbf{x} \rrbracket /\left(\mathbf{f}^{\left[p^{n}\right]}\right) \llbracket \mathbf{y} \rrbracket \rightarrow k \llbracket \mathbf{x} \rrbracket /\left(\mathbf{f}^{p^{n}}\right)$ which leaves the elements of $k$ fixed and such that $\rho_{R}\left(x_{i}+\left(\mathbf{f}^{\left[p^{n}\right]}\right)\right)=x_{i}^{p^{n}}+\left(\mathbf{f}^{p^{n}}\right)$ and $\rho_{R}\left(y_{i}+\left(\mathbf{f}^{\left[p^{n}\right]}\right)\right)=$ $x_{i}+\left(\mathbf{f}^{p^{n}}\right)$. Next, we have the natural surjection $\phi_{R}^{\prime \prime}: k \llbracket \mathbf{x} \rrbracket /\left(\mathbf{f}^{p^{n}}\right) \rightarrow k \llbracket \mathbf{x} \rrbracket /(\mathbf{f})$. As is observed in the proof of [9, Proposition 12.2.7], $\operatorname{Ker}\left(\rho_{R}\right)$ is generated by the sequence $x_{1}-y_{1}^{p^{n}}, \ldots, x_{e}-y_{e}^{p^{n}}$. The factorization of $f_{S}^{n}$ is defined similarly in terms of $\dot{\varphi}_{S}, \rho_{S}$, and $\phi_{S}^{\prime \prime}$.

This provides the outer edges of the following commutative diagram:

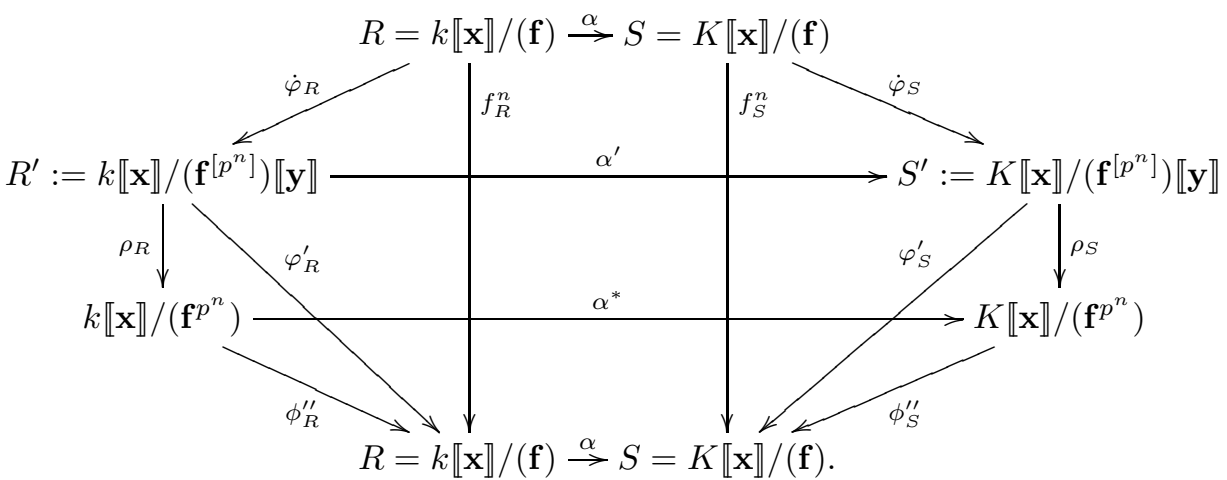

The maps $\alpha^{\prime}$ and $\alpha^{*}$ are induced by the inclusion $k \subseteq K$. Hence, they are weakly étale, by the same proof as for $\alpha$.

Next, we verify the pushout condition. Since $\phi_{R}^{\prime \prime} \rho_{R}$ and $\phi_{S}^{\prime \prime} \rho_{S}$ are surjective and $\alpha^{\prime}$ is flat, it suffices to show that $\operatorname{Ker}\left(\phi_{S}^{\prime \prime} \rho_{S}\right)$ is generated by the images in $K \llbracket \mathbf{x} \rrbracket /\left(\mathbf{f}^{\left[p^{n}\right]}\right) \llbracket \mathbf{y} \rrbracket$ of the generators of $\operatorname{Ker}\left(\phi_{R}^{\prime \prime} \rho_{R}\right)$. From the above discussion, we know that $\operatorname{Ker}\left(\rho_{R}\right)$ is generated by the sequence $x_{1}-y_{1}^{p^{n}}, \ldots, x_{e}-y_{e}^{p^{n}}$. Since $\rho_{S}$ is 
constructed exactly like $\rho_{R}$, we conclude that $\operatorname{Ker}\left(\rho_{S}\right)$ is generated by the sequence $x_{1}-y_{1}^{p^{n}}, \ldots, x_{e}-y_{e}^{p^{n}}$. Also, by construction, $\operatorname{Ker}\left(\phi_{R}^{\prime \prime}\right)$ and $\operatorname{Ker}\left(\phi_{S}^{\prime \prime}\right)$ are generated by the images of $\mathbf{f}$ in the respective rings. Thus, $\operatorname{Ker}\left(\phi_{R}^{\prime \prime} \rho_{R}\right)$ and $\operatorname{Ker}\left(\phi_{S}^{\prime \prime} \rho_{S}\right)$ are both generated by $x_{1}-y_{1}^{p^{n}}, \ldots, x_{e}-y_{e}^{p^{n}}, \mathbf{f}$ as desired.

Finally, if $K$ is $F$-finite, then $S$ and $S^{\prime}$ are $F$-finite by [25, Corollary 2.6].

The next result is like Theorem 3.8

Theorem 4.2. Let $(R, \mathfrak{m}, k)$ be a local ring of prime characteristic $p>0$, and let $C$ be a semidualizing $R$-complex. Then the following are equivalent:

(i) $C$ is a dualizing $R$-complex.

(i') There is a complete weakly étale $F$-finite local $R$-algebra $S$ such that $S \otimes_{R}^{\mathbf{L}} C$ is dualizing for $S$.

(ii) $\widehat{R} \otimes_{R}^{\mathbf{L}} C \sim\left(\widehat{R} \otimes_{R}^{\mathbf{L}} C\right)\left(\widehat{f}_{R}^{n}\right)$ for some $n>0$.

(ii') There is a complete weakly étale $F$-finite local $R$-algebra $S$ such that $S \otimes{ }_{R}^{\mathbf{L}} C \sim$ $\left(S \otimes_{R}^{\mathbf{L}} C\right)\left(f_{S}^{n}\right)$ for some $n>0$.

(iii) $\mathrm{G}_{C}$-dim $f_{R}^{n}<\infty$ and $\widehat{R} \otimes_{R}^{\mathbf{L}} C$ is derived $\left(\widehat{R} \otimes \otimes_{R}^{\mathbf{L}} C\right)\left(f_{\widehat{R}}^{n}\right)$-reflexive for some $n>0$.

(iii') There is a complete weakly étale $F$-finite local $R$-algebra $S$ such that for some $n>0$ we have $\mathrm{G}_{S \otimes_{R}^{\mathrm{L}} C}-\operatorname{dim} f_{S}^{n}<\infty$ and $S \otimes_{R}^{\mathrm{L}} C$ is derived $\left(S \otimes_{R}^{\mathrm{L}} C\right)\left(f_{S}^{n}\right)$ reflexive.

(iv) $\mathrm{G}_{C}-\operatorname{dim} f_{R}^{n}<\infty$ for infinitely many $n>0$.

(iv') There is a complete weakly étale $F$-finite local $R$-algebra $S$ such that for infinitely many $n>0$ we have $\mathrm{G}_{S \otimes_{R}^{\mathrm{L} C}}-\operatorname{dim} f_{S}^{n}<\infty$.

(v') There is a complete weakly étale $F$-finite local $R$-algebra $S$ such that for some $n>0$ we have $\mathrm{G}_{S \otimes_{R} \mathbf{L} C}-\operatorname{dim} f_{S}^{n}<\infty$ and ${ }^{n} S \otimes_{S}^{L} \mathbf{R} \operatorname{Hom}_{S}\left(S \otimes_{R}^{\mathbf{L}} C, D^{S}\right)$ is derived $\mathbf{R H o m}_{S}\left(S \otimes_{R}^{\mathbf{L}} C, D^{S}\right)$-reflexive, where $D^{S}$ is a dualizing $S$-complex.

If $R$ has a dualizing complex $D$, then these conditions are equivalent to the following:

(v) $\mathrm{G}_{C}$ - $\operatorname{dim} f_{R}^{n}<\infty$ and ${ }^{n} R \otimes_{R}^{L} C^{\dagger_{D}}$ is derived $C^{\dagger D}$-reflexive for some $n>0$.

Proof. The proof is like that of Theorem 3.8. The only difference is in the equivalences (ii) $\Longleftrightarrow$ (ii') and (iii) $\Longleftrightarrow$ (iii'), in which we use Lemma 4.1, where $K$ is an algebraic closure of $k$.

The last result of this section is proved like Theorem 3.15

Theorem 4.3. Assume that $R$ is a local ring of prime characteristic $p>0$, and let $C$ be a semidualizing $R$-complex. Then the following conditions are equivalent:

(i) $R$ is Gorenstein.

(ii) $\mathrm{G}_{C}$-dim $f_{R}^{m}<\infty$ for all $m>0$, and ${ }^{n} R \in \mathcal{B}_{C}(R)$ for all $n>0$.

(ii') For every complete weakly étale $F$-finite local $R$-algebra $S$, one has ${ }^{n} S \in$ $\mathcal{B}_{S \otimes_{R} \mathrm{~L} C}(S)$ for all $n>0$, and $\mathrm{G}_{S \otimes_{R} C^{-}} \operatorname{dim} f_{S}^{m}<\infty$ for all $m>0$.

(iii) $\mathrm{G}_{C}$-dim $f_{R}^{m}<\infty$ for infinitely many $m>0$, and ${ }^{n} R \in \mathcal{B}_{C}(R)$ for some $n>0$.

(iii') There is a complete weakly étale $F$-finite local $R$-algebra $S$ such that ${ }^{n} S \in$ $\mathcal{B}_{S \otimes_{R}^{\mathrm{L}} C}(S)$ for some $n>0$, and $\mathrm{G}_{S \otimes_{R}^{\mathrm{L}} C^{-}} \operatorname{dim} f_{S}^{m}<\infty$ for infinitely many $m>0$.

(iv) $\mathrm{G}_{C}$-dim $f_{R}^{m}<\infty$ for some $m>0$, and ${ }^{n} R \in \mathcal{B}_{C}(R)$ for infinitely many $n>0$.

(iv') There is a complete weakly étale $F$-finite local $R$-algebra $S$ such that ${ }^{n} S \in$ $\mathcal{B}_{S \otimes_{R}^{\mathrm{L}} C}(S)$ for infinitely many $n>0$, and $\mathrm{G}_{S \otimes_{R}^{\mathrm{L} C}}-\operatorname{dim} f_{S}^{m}<\infty$ for some $m>0$. 


\section{Appendix A. A Construction of Endomorphisms}

The point of this section is found in Proposition A.3, which guarantees the existence of non-trivial cows diagrams (3.5.1); see Remark 3.6.

Lemma A.1. Let $\alpha: k \rightarrow k$ be a field endomorphism. Then there is a commutative diagram of field extensions

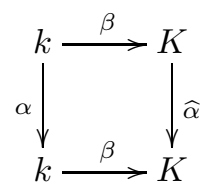

such that $\widehat{\alpha}$ is an isomorphism. Moreover, if $\alpha$ is separable, then so is $\beta$.

Proof. Let $K$ be the direct limit in the category of fields and field extensions of the directed system $k \stackrel{\alpha}{\rightarrow} k \stackrel{\alpha}{\rightarrow} k \stackrel{\alpha}{\rightarrow} \cdots$. The universal mapping property for direct limits provides a unique field endomorphism $\widehat{\alpha}: K \rightarrow K$ making the following diagram commute

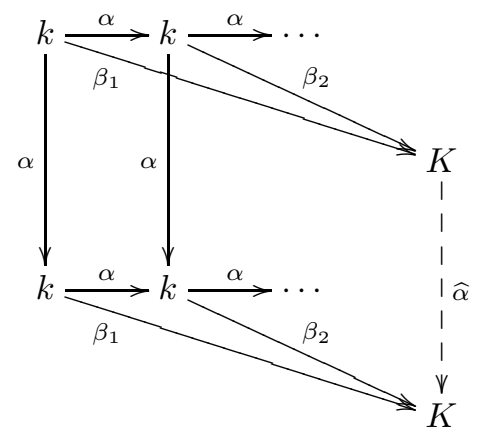

where the maps $\beta_{i}$ are the universal ones for $K$. With $\beta:=\beta_{1}$, we have the commutativity of (A.1.1), so we need to show that $\widehat{\alpha}$ is an isomorphism. Since $\widehat{\alpha}$ is a morphism of fields, it is injective, so we need only verify surjectivity. For this, let $x \in K=\cup_{i=1}^{\infty} \operatorname{Im}\left(\beta_{i}\right)$. Then there is an index $i$ and an element $y \in k$ such that

$$
x=\beta_{i}(y)=\beta_{i+1}(\alpha(y))=\widehat{\alpha}\left(\beta_{i+1}(y)\right) \in \operatorname{Im}(\widehat{\alpha}) .
$$

this yields the surjectivity of $\widehat{\alpha}$.

To complete the proof, assume that $\alpha$ is separable. It follows that $\alpha^{n}$ is separable for each $n \geqslant 1$. To show that $\beta$ is separable, we need to show that for every intermediate field $k \rightarrow F \rightarrow K$ such that $F$ is finitely generated as a field extension of $k$, the extension $k \rightarrow F$ is separably generated; see [22, Theorem VI.2.10]. Again, $K$ is the union of the images of the $\beta_{i}$. The finite generation condition on $F$ implies that the generators for $F$ over $k$ lie in some "finite stage" of the limit. In other words, the commutative diagram

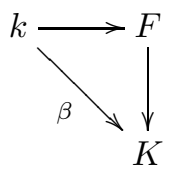


gives rise to another commutative diagram

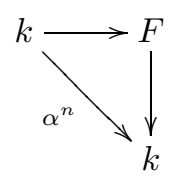

for some $n \geqslant 1$. Since $\alpha^{n}$ is separable, the intermediate extension $k \rightarrow F$ is separably generated, as desired.

The next result takes a construction of Grothendieck [20, Proposition (0.10.3.1)] and manipulates it a bit, similarly to Lemma 4.1 .

Lemma A.2. Let $\varphi:(R, \mathfrak{m}, k) \rightarrow(R, \mathfrak{m}, k)$ be a contracting endomorphism, and consider a commutative diagram of field extensions

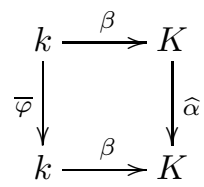

where $\bar{\varphi}$ is the map induced by $\varphi$. Assume that $\beta$ is separable. Then there is a commutative diagram of local ring homomorphisms

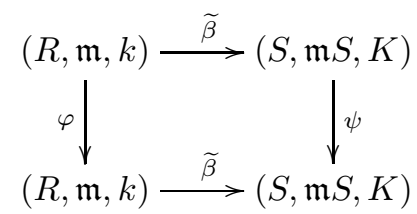

such that $S$ is complete, $\widetilde{\beta}$ is weakly étale, $\psi$ is a contracting endomorphism, and the diagram induced by (A.2.2) on residue fields is (A.2.1).

Proof. By [20, Proposition (0.10.3.1)] there is a weakly étale local ring homomorphism $\widetilde{\beta}:(R, \mathfrak{m}, k) \rightarrow(S, \mathfrak{m} S, K)$. Replace $S$ with its completion if necessary to assume that $S$ is complete. Since the induced map $\beta: k \rightarrow K$ is separable, we conclude that $\widetilde{\beta}$ is formally smooth. Since $S$ is complete, a standard application of smoothness provides a local ring homomorphism $\psi: S \rightarrow S$ such that (1) $\psi$ induces $\widehat{\alpha}$ on residue fields, and (2) $\psi$ respects the $R$-algebra structures given by $\widetilde{\beta}$ and $\widetilde{\beta} \varphi$, that is, such that the diagram A.2.2 commutes.

It remains to show that $\psi$ is contracting. For this, fix an integer $i \geqslant 1$ such that $\varphi^{i}(\mathfrak{m}) \subseteq \mathfrak{m}^{2}$. Then

$$
\psi^{i}(\mathfrak{m} S) \subseteq \psi^{i}(\mathfrak{m} S) S=\psi^{i}(\widetilde{\beta}(\mathfrak{m})) S=\widetilde{\beta}\left(\varphi^{i}(\mathfrak{m})\right) S \subseteq \widetilde{\beta}\left(\mathfrak{m}^{2}\right) S=(\mathfrak{m} S)^{2}
$$

as desired.

Proposition A.3. Let $\varphi:(R, \mathfrak{m}, k) \rightarrow(R, \mathfrak{m}, k)$ be a contracting endomorphism such that $R / \varphi(\mathfrak{m}) R$ is artinian and the induced map $\bar{\varphi}: k \rightarrow k$ is separable. Then 
there is a commutative diagram of local ring homomorphisms

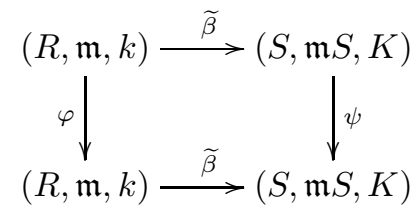

such that $\widetilde{\beta}$ is weakly étale and the induced map $k \rightarrow K$ is separable (hence $\widetilde{\beta}$ is formally smooth), $S$ is complete, and $\psi$ is a module-finite contracting endomorphism.

Proof. Lemma A.1 provides a commutative diagram of field extensions

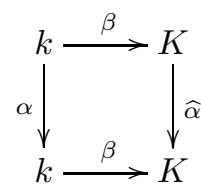

such that $\widehat{\alpha}$ is an isomorphism and $\beta$ is separable. Hence, Lemma A.2 implies that we have a commutative diagram of local ring homomorphisms

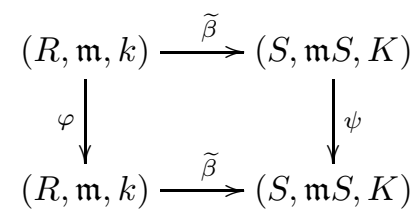

such that $\widetilde{\beta}$ is weakly étale, $\psi$ is a contracting endomorphism, and the diagram induced by (A.3.3 on residue fields is A.3.2. To avoid ambiguous notation, given an $S$-module $M$, let ${ }^{\psi} M$ denote the $S$-module structure on $M$ given by restriction of scalars along $\psi$.

It remains to show that $\psi$ is module-finite. Since $S$ is complete and local, the complete Nakayama's Lemma [26, Theorem 8.4] says that it suffices to show that $\psi(S / \psi(\mathfrak{m} S) S)$ has finite length over $S$. Since the map $\widehat{\alpha}: S / \mathfrak{m} S \rightarrow S / \mathfrak{m} S$ induced by $\psi$ is an isomorphism, if $M$ is an $S$-module of finite length, then ${ }^{\psi} M$ also has finite length; in fact, the lengths are the same in this case. Thus, it remains to show that $S / \psi(\mathfrak{m} S) S$ has finite length over $S$.

By assumption, the quotient ring $R / \varphi(\mathfrak{m}) R$ is artinian. This means that $\mathfrak{m}^{n} \subseteq$ $\varphi(\mathfrak{m}) R$ for $n \gg 0$, so

$$
(\mathfrak{m} S)^{n}=\widetilde{\beta}\left(\mathfrak{m}^{n}\right) S \subseteq \widetilde{\beta}(\varphi(\mathfrak{m})) S=\psi(\widetilde{\beta}(\mathfrak{m})) S=\psi(\mathfrak{m} S) S .
$$

We conclude that $S / \psi(\mathfrak{m} S) S$ is artinian, so it has finite length over $S$.

\section{ACKNOWLEDGMENTS}

We are grateful to Hamid Rahmati for pointing out [8, Remark 5.9].

\section{REFERENCES}

1. M. André, Homologie des algèbres commutatives, Springer-Verlag, Berlin, 1974, Die Grundlehren der mathematischen Wissenschaften, Band 206. MR 0352220 (50 \#4707)

2. M. Auslander and M. Bridger, Stable module theory, Memoirs of the American Mathematical Society, No. 94, American Mathematical Society, Providence, R.I., 1969. MR 42 \#4580 
3. M. Auslander and D. A. Buchsbaum, Homological dimension in local rings, Trans. Amer. Math. Soc. 85 (1957), 390-405. MR 0086822 (19,249d)

4. L. L. Avramov and H.-B. Foxby, Homological dimensions of unbounded complexes, J. Pure Appl. Algebra 71 (1991), 129-155. MR 93g:18017

5. L Locally Gorenstein homomorphisms, Amer. J. Math. 114 (1992), no. 5, 1007-1047. MR 1183530 (93i:13019)

6. _ Ring homomorphisms and finite Gorenstein dimension, Proc. London Math. Soc. (3) 75 (1997), no. 2, 241-270. MR 98d:13014

7. L. L. Avramov, H.-B. Foxby, and B. Herzog, Structure of local homomorphisms, J. Algebra 164 (1994), 124-145. MR 95f:13029

8. L. L. Avramov, M. Hochster, S. B. Iyengar, and Y. Yao, Homological invariants of modules over contracting endomorphisms, preprint (2011), arXiv:1010.3029v3.

9. L. L. Avramov, S. Iyengar, and C. Miller, Homology over local homomorphisms, Amer. J. Math. 128 (2006), no. 1, 23-90. MR 2197067

10. L. L. Avramov, S. B. Iyengar, and J. Lipman, Reflexivity and rigidity for complexes. I. Commutative rings, Algebra Number Theory 4 (2010), no. 1, 47-86. MR 2592013

11. L. W. Christensen, Semi-dualizing complexes and their Auslander categories, Trans. Amer. Math. Soc. 353 (2001), no. 5, 1839-1883. MR 2002a:13017

12. L. W. Christensen, A. Frankild, and H. Holm, On Gorenstein projective, injective and flat dimensions - a functorial description with applications, J. Algebra 302 (2006), no. 1, 231-279. MR 2236602

13. L. W. Christensen and H. Holm, Ascent properties of Auslander categories, Canad. J. Math. 61 (2009), no. 1, 76-108. MR 2488450

14. H.-B. Foxby, Isomorphisms between complexes with applications to the homological theory of modules, Math. Scand. 40 (1977), no. 1, 5-19. MR 0447269 (56 \#5584)

15. H.-B. Foxby and A. J. Frankild, Cyclic modules of finite Gorenstein injective dimension and Gorenstein rings, Illinois J. Math. 51 (2007), no. 1, 67-82 (electronic). MR 2346187 (2008i:13022)

16. H.-B. Foxby and A. Thorup, Minimal injective resolutions under flat base change, Proc. Amer. Math. Soc. 67 (1977), no. 1, 27-31. MR 56 \#11984

17. A. Frankild and S. Sather-Wagstaff, Reflexivity and ring homomorphisms of finite flat dimension, Comm. Algebra 35 (2007), no. 2, 461-500. MR 2294611

18. S. I. Gelfand and Y. I. Manin, Methods of homological algebra, Springer-Verlag, Berlin, 1996. MR 2003m:18001

19. S. Goto, A problem on Noetherian local rings of characteristic p, Proc. Amer. Math. Soc. 64 (1977), no. 2, 199-205. MR 0447212 (56 \#5527)

20. A. Grothendieck, Éléments de géométrie algébrique. III. Étude cohomologique des faisceaux cohérents. I, Inst. Hautes Études Sci. Publ. Math. (1961), no. 11, 167. MR 0217085 (36 \#177c)

21. R. Hartshorne, Residues and duality, Lecture Notes in Mathematics, No. 20, Springer-Verlag, Berlin, 1966. MR 36 \#5145

22. T. W. Hungerford, Algebra, Graduate Texts in Mathematics, vol. 73, Springer-Verlag, New York, 1980, Reprint of the 1974 original. MR 600654 (82a:00006)

23. S. Iyengar and S. Sather-Wagstaff, G-dimension over local homomorphisms. Applications to the Frobenius endomorphism, Illinois J. Math. 48 (2004), no. 1, 241-272. MR 2048224 (2005c:13016)

24. E. Kunz, Characterizations of regular local rings of characteristic p, Amer. J. Math. 91 (1969), 772-784. MR 0252389 (40 \#5609)

25. E. Kunz, On Noetherian rings of characteristic p, Amer. J. Math. 98 (1976), no. 4, 999-1013. MR 0432625 (55 \#5612)

26. H. Matsumura, Commutative ring theory, second ed., Studies in Advanced Mathematics, vol. 8, University Press, Cambridge, 1989. MR 90i:13001

27. S. Nasseh and S. Sather-Wagstaff, Cohen factorizations: weak functoriality and applications, preprint (2012) arXiv:1205.2119.

28. S. Nasseh, M. Tousi, and S. Yassemi, Characterization of modules of finite projective dimension via Frobenius functors, Manuscripta Math. 130 (2009), no. 4, 425-431. MR 2563144 (2010j:13028) 
29. A. G. Rodicio, On a result of Avramov, Manuscripta Math. 62 (1988), no. 2, 181-185. MR 89k:13014

30. S. Sather-Wagstaff, Complete intersection dimensions and Foxby classes, J. Pure Appl. Algebra 212 (2008), no. 12, 2594-2611. MR 2452313 (2009h:13015)

31. B Bass numbers and semidualizing complexes, Commutative algebra and its applications, Walter de Gruyter, Berlin, 2009, pp. 349-381. MR 2640315

32. J.-P. Serre, Sur la dimension homologique des anneaux et des modules noethériens, Proceedings of the international symposium on algebraic number theory, Tokyo \& Nikko, 1955 (Tokyo), Science Council of Japan, 1956, pp. 175-189. MR 19,119a

33. R. Takahashi and Y. Yoshino, Characterizing Cohen-Macaulay local rings by Frobenius maps, Proc. Amer. Math. Soc. 132 (2004), no. 11, 3177-3187. MR 2073291 (2005d:13008)

34. J.-L. Verdier, Catégories dérivées, SGA $4 \frac{1}{2}$, Springer-Verlag, Berlin, 1977, Lecture Notes in Mathematics, Vol. 569, pp. 262-311. MR 57 \#3132

35. _ Des catégories dérivées des catégories abéliennes, Astérisque (1996), no. 239, xii+253 pp. (1997), With a preface by Luc Illusie, Edited and with a note by Georges Maltsiniotis. MR 98c:18007

36. S. Yassemi, G-dimension, Math. Scand. 77 (1995), no. 2, 161-174. MR 97d:13017

Mathematics Department, NDSU Dept 2750, PO Box 6050, Fargo, ND 58108-6050 USA

E-mail address: saeed.nasseh@ndsu.edu

$U R L:$ http://www.ndsu.edu/pubweb/〜nasseh/

E-mail address: sean.sather-wagstaff@ndsu.edu

$U R L:$ http://www.ndsu.edu/pubweb/ ${ }^{\text {ssatherw/ }}$ 